\title{
Pythagorean Fuzzy Soft Einstein Ordered Weighted Average Operator in Sustainable Supplier Selection Problem
}

\author{
Rana Muhammad Zulqarnain $\left(\mathbb{D},{ }^{1}\right.$ Imran Siddique $\mathbb{D}^{\mathrm{D}},{ }^{2}$ Shahzad Ahmad, ${ }^{1}$ \\ Aiyared Iampan $\left(\mathbb{D},{ }^{3}\right.$ Goran Jovanov $\mathbb{D}^{4},{ }^{4}$ Đorde Vranješ $\left(\mathbb{D},{ }^{5}\right.$ and Jovica Vasiljevićc \\ ${ }^{1}$ Department of Mathematics, School of Science, University of Management and Technology, Sialkot Campus, Lahore, Pakistan \\ ${ }^{2}$ Department of Mathematics, School of Science, University of Management and Technology, Lahore 54770, Pakistan \\ ${ }^{3}$ Department of Mathematics, School of Science, University of Phayao, Mae Ka, Mueang, Phayao 56000, Thailand \\ ${ }^{4}$ University of Criminal Investigation and Police Studies, Department of Forensics, Cara Dušana 196, 11080 Belgrade, Serbia \\ ${ }^{5}$ Environ, D.O.O, Čukarička 9, Belgrade, Serbia \\ ${ }^{6}$ City Secretary, City Administration of Belgrade, Secretariat for Public Transport, 43-45 27 Marta Street, 11000 Belgrade, Serbia
}

Correspondence should be addressed to Imran Siddique; imransiddique@umt.edu.pk and Đorđe Vranješ; djordjevranjes@yahoo.com

Received 5 October 2021; Revised 30 October 2021; Accepted 2 November 2021; Published 30 November 2021

Academic Editor: Muhammet Deveci

Copyright (c) 2021 Rana Muhammad Zulqarnain et al. This is an open access article distributed under the Creative Commons Attribution License, which permits unrestricted use, distribution, and reproduction in any medium, provided the original work is properly cited.

\begin{abstract}
Pythagorean fuzzy soft set (PFSS) is the most influential and operative extension of the Pythagorean fuzzy set (PFS), which contracts with the parametrized standards of the substitutes. It is also a generalized form of the intuitionistic fuzzy soft set (IFSS) and delivers a well and accurate estimation in the decision-making (DM) procedure. The primary purpose is to prolong and propose ideas related to Einstein's ordered weighted aggregation operator from fuzzy to PFSS, comforting the condition that the sum of the degrees of membership function and nonmembership function is less than one and the sum of the squares of the degree of membership function and nonmembership function is less than one. We present a novel Pythagorean fuzzy soft Einstein ordered weighted averaging (PFSEOWA) operator based on operational laws for Pythagorean fuzzy soft numbers. Furthermore, some essential properties such as idempotency, boundedness, and homogeneity for the proposed operator have been presented in detail. The choice of a sustainable supplier is also examined as an essential part of sustainable supply chain management (SSCM) and is considered a crucial multiattribute group decisionmaking (MAGDM) issue. In some MAGDM problems, the relationship between alternatives and uncertain environments will be the main reason for deficient consequences. We have presented a novel aggregation operator for PFSS information to choose sustainable suppliers to cope with those complex issues. The Pythagorean fuzzy soft number (PFSN) helps to represent the obscure information in such real-world perspectives. The priority relationship of PFSS details is beneficial in coping with SSCM. The proposed method's effectiveness is proved by comparing advantages, effectiveness, and flexibility among the existing studies.
\end{abstract}

\section{Introduction}

Decision-making is a preconceived strategy of picking a logical choice between many objects. Decision-making (DM) plays a crucial part in real-life scenarios. Better decision-making will change the process to determine the limits, benefits, and characteristics of the decision-maker. To cope with the designated scenario, Zaheh [1] launched the fuzzy set (FS) paradigm that puts advancement in several fields of science and technology, nominating the membership grade for each object real values among 0 and 1 . In conventional set theory, elements of a set can be either 0 or 1, but in FS, the degree of membership ranges from 0 to 1 . Atanassov [2] extended the concept of FS and introduced an intuitionistic fuzzy set (IFS) which considered both membership and nonmembership grades. Zeshui Xu [3] presented some novel aggregation operators (AOs) for IFS and utilized their developed operators for DM. Wang and Liu [4] offered intuitionistic fuzzy Einstein weighted geometric and intuitionistic fuzzy Einstein ordered weighted geometric 
operators with desirable properties. They also constructed multiple attribute decision-making (MADM) techniques based on their developed operators. Atanassov and Gargov [5] prolonged the notion of IFS and established the concept of the interval-valued intuitionistic fuzzy set with some novel operations and their characteristics.

IFS is a powerful concept, which various researchers have studied since its development. However, the dominant concept of IFS has some shortcomings, such as degree of membership and nonmembership taken so that their sum exceeds 1. To cope with these limitations, IFS fails to overcome the scenarios mentioned above. Yager [6] extended the notion of IFS and developed the Pythagorean fuzzy set (PFS) by amending the condition $\mathrm{MG}+\mathrm{NMG} \leq 1$ to $\mathrm{MG}^{2}+\mathrm{NMG}^{2} \leq 1$. Rehman et al. [7] developed the Pythagorean fuzzy weighted averaging aggregation operator with fundamental properties and offered a DM method based on their developed operator. Pamučar and Savin [8] established the best worst method and the compressed proportional assessment models for the assortment of the optimum off-road vehicle. Rehman et al. [9] presented a Pythagorean fuzzy ordered weighted averaging aggregation operator with desirable properties and constructed a MADM approach for the developed operator. Wang and $\mathrm{Li}$ [10] planned Bonferroni mean AOs for PFS and built the MADM method utilizing their settled operators. Deveci et al. [11] presented a comprehensive survey to justify the operations and properties for PFS. Garg [12] developed Pythagorean fuzzy geometric interactive AOs based on Einstein operations with their essential properties. Ali et al. [13] proposed the Einstein operational laws utilizing $t$-norm and $t$-conorm for complex interval-valued PFS. Alosta et al. [14] utilized the multicriteria decision-making technique to enhance emergency medical service centers' finest sites. Milosevic et al. [15] constructed a novel model operating fuzzy logic systems to select a route for the transportation of harmful ingredients.

Above mentioned theories and their corresponding DM approaches are acknowledged and utilized by experts in several fields. Still, due to the lack of parametrized values, these approaches cannot solve parametrization problems. Molodtsov [16] presented the solution of vagueness and uncertainty, introduced a soft set (SS), and discussed some basic operations with their properties. Maji et al. [17] extended the concept of SS and defined numerous basic operations with their essential features and operated to solve DM [18] complications. Maji et al. [19] protracted the notion of FSS and introduced the IFSS with some fundamental operations. Zulqarnain et al. [20] established the correlation coefficient (CC) for interval-valued IFSS and operated their settled CC for the structure of the TOPSIS method. Zulqarnain and Dayan [21] employed the intuitionistic fuzzy TOPSIS for the assortment of an autocorporation. Muhammad Zulqarnain et al. [22] protracted the idea of the IFSS to an intuitionistic fuzzy hypersoft set and presented the TOPSIS method based on the CC. Garg and Arora [23] planned the generalized AOs for the IFSS.

Several investigators prolonged the SS concept utilizing the fundamental definition of FSS. Peng et al. [24] proposed the impression of IFSS to PFSS by modifying the condition $\mathrm{MG}+\mathrm{NMG} \leq 1$ to $\mathrm{MG}^{2}+\mathrm{NMG}^{2} \leq 1$ with some desirable operations. Athira et al. [25] utilized the Hamming distance and Euclidean distance to propose the entropy measure for PFSS. Athira et al. [26] established a DM technique using distance-based entropy measures to resolve DM complications for PFSS. Naeem et al. [27] considered the linguistic PFSS and introduced some basic operations with their properties for PFSS. They also proposed the TOPSIS and VIKOR methods under considered environment to solve DM issues. Riaz et al. [28] prolonged the notion of m-polar PFSS and offered the TOPSIS technique for $m$ polar PFSS to resolve multicriteria group decision-making (MCGDM) problems. Riaz et al. [29] anticipated the similarity measures for PFSS and constructed a DM method for PFSS using their developed similarity measures. Zulqarnain et al. [30] settled the AOs for PFSS and projected a DM procedure based on their developed operators. Zulqarnain et al. [31] prolonged the TOPSIS method for PFSS based on CC and employed their progressive approach for MADM problems. Zulqarnain et al. [32] introduced some novel operational laws considering the interaction and proposed interactive AOs for PFSS. They also developed the MCDM approach utilizing their established interactive AOs. Siddique et al. [33] acquired some algebraic operations for PFSS and built a DM technique for PFSS based on a score matrix. It has been observed that fuzzy numbers can only measure uncertainty, and intuitionistic fuzzy numbers can measure true and false membership values. The sum of true and false membership values must be less than 1. However, in our developed methodology, we can measure the values of truth and false membership by modifying the intuitionistic fuzzy numbers condition, such as the sum of the square of true and false values must be less or equal to 1 .

Selection and evaluation of suppliers are essential features in professional activities. The fluctuations of the current government strategy use supplier classification as measured by multiple theories with environmental and social needs. Therefore, in the literature, the issue is called sustainable supplier selection, a reference issue of MCGDM. At the same time, multiple credentials [34-37] point to the need for further study through the MCDM approach in supplier selection, focusing on appropriate glossary considerations on environmental realities and expert predictions. To solve such shortcomings, we have implemented a method of choosing sustainable suppliers with Pythagorean fuzzy soft information. The stimulation reassessment is considered by utilizing Pythagorean fuzzy soft numbers. The PFSN is helpful to comply with imprecise information in everyday life complications. In the prevailing literature [38, 39], numerous Einstein AOs have been familiarized, such as Pythagorean fuzzy Einstein weighted average, Einstein weighted geometric, Einstein ordered weighted average, and Einstein ordered weighted geometric operators, to solve the complex problems of DM. The Einstein weighted AOs only weight the Pythagorean fuzzy argument. At the same time, the Einstein weighted ordered AOs only weight the orderly position of the Pythagorean fuzzy argument not the Pythagorean fuzzy argument itself. These Einstein 
ordered operators for PFS are unable to accommodate the parametrization values of the alternatives. To overcome the drawbacks mentioned above, we focus on developing some novel Einstein AOs for PFSS.

Thus, the current work intends to offer a novel PFSEOWA operator. It is expected to follow the proposed operator's algorithm rules to solve the DM problem and numerical examples used to prove the effectiveness of the introduced DM method. The proposed operators' key benefit is that the proposed operators can reduce IFSS and FSS operators under specific confident limitations. The rest of the research is organized as follows: some fundamental concepts like FS, IFS, PFS, SS, FSS, IFSS, and PFSS are discussed in Section 2. In Section 3, we developed the PFSEOWA operator. Some desired properties of proposed operators also have been discussed in Section 3. Section 4 developed the MAGDM approach based on proposed operators and presented a numerical example of SSCM. In Section 5, a comparison with some existing methodologies has been provided.

\section{Preliminaries}

This section comprises some basic definitions such as SS, IFS, PFS, IFSS, and PFSS, which will provide a foundation to form the structure of the following manuscript.

Definition 1 (see [16]). Let $X$ be a universal set and $\mathbb{N}=$ $\left\{t_{1}, t_{2}, t_{3}, \ldots, t_{m}\right\}$ be the set of attributes, then a pair $(\Omega, \mathbb{N})$ is called a soft set (SS) over $X$ where $\Omega: \mathbb{N} \longrightarrow K^{X}$ is a mapping and $K^{X}$ is known as a collection of all subsets of universal set $X$.

Definition 2 (see [6]). Let $X$ be a collection of objects, then a PFS $A$ over $X$ is defined as

$$
A=\left\{\left(t, \mathfrak{a}_{A}(t), \mathfrak{b}_{A}(t)\right) \mid t \in X\right\},
$$

where $\mathfrak{a}_{A}(t), \mathfrak{b}_{A}(t): X \longrightarrow[0,1]$ represent the membership and nonmembership grade functions, respectively. Furthermore, $0 \leq \mathfrak{a}_{A}(t)^{2}+\mathfrak{b}_{A}(t)^{2} \leq 1$ and $I=1-\mathfrak{a}(t)^{2}-\mathfrak{b}_{A}(t)^{2}$ is called degree of indeterminacy.

We can see from the above definitions that the only difference is in the conditions, i.e., in IFS, we deal with the state $\quad 0 \leq \mathfrak{a}_{A}(t)+\mathfrak{b}_{A}(t) \leq 1$ and $\quad I=1-\mathfrak{a}_{A}(t)-\mathfrak{b}_{A}(t)$ whereas in PFS, we have condition $0 \leq \mathfrak{a}_{A}(t)^{2}+\mathfrak{b}_{A}(t)^{2} \leq 1$ and $I=1-\mathfrak{a}_{A}(t)^{2}-\mathfrak{b}_{A}(t)^{2}$. We can say that a PFS is the general case of IFS.

Definition 3 (see [24]). Let $X$ be a universal set and $\mathbb{N}$ be set of attributes, then a pair $(\Omega, \mathbb{N})$ is called an IFSS over $X$ where $\Omega: \mathbb{N} \longrightarrow I K^{X}$ is a mapping and $I K^{X}$ is known as a collection of all IFS subsets of universal set $X$.

$$
(\Omega, A)=\left\{t,\left(\mathfrak{a}_{A}(t), \mathfrak{b}_{A}(t)\right) \mid t \in A\right\},
$$

where $\mathfrak{a}_{A}(t), \mathfrak{b}_{A}(t): A \longrightarrow[0,1]$ are membership grade and nonmembership functions, respectively, with $0 \leq \mathfrak{a}_{A}(t)+\mathfrak{b}_{A}(t) \leq 1$ and $A \subset \mathbb{N}$.
Definition 4 (see [24]). Let $(\Omega, A)$ and $(\Omega, B)$ be two IFSS. Then, some basic operations for IFSS are defined as follows:

(1) $(\Omega, A)$ is said to be an intuitionistic fuzzy soft subset of $(\Omega, B)$. If and $A \subset B$ and $\mathfrak{a}_{A}(t) \leq \mathfrak{a}_{B}(t)$, $\mathfrak{b}_{A}(t) \geq \mathfrak{b}_{B}(t)$ for all $t \in A$.

(2) Complement of $(\Omega, A)$ is denoted by $\left(\Omega, A^{c}\right)$ and is defined as

$$
\left(\Omega, A^{c}\right)=\left\{t,\left(\mathfrak{b}_{A}(t), \mathfrak{a}_{A}(t)\right) \mid t \in A\right\} .
$$

(3) Union of two IFSSs is defined as follows:

$$
(\Omega, A) \cup(\Omega, B)=\left\{t, \max \left(\mathfrak{a}_{A}(t), \mathfrak{a}_{B}(t)\right) \mid \forall t \in A\right\} .
$$

(4) The intersection of $(\Omega, A)$ and $(\Omega, B)$ can be defined as follows:

$$
(\Omega, A) \cap(\Omega, B)=\left\{t, \min \left(\mathfrak{a}_{A}(t), \mathfrak{a}_{B}(t)\right) \mid \forall t \in A\right\} .
$$

Definition 5 (see [24]). Let $X$ be a universal set and $\mathbb{N}$ be set of attributes, then a pair $(\Omega, \mathbb{N})$ is called a PFSS over $X$ where $\Omega: \mathbb{N} \longrightarrow \wp K^{X}$ is a mapping and $\wp K^{X}$ is known as the collection of all PFS subsets of universal set $X$.

$$
(\Omega, A)=\left\{t,\left(\mathfrak{a}_{A}(t), \mathfrak{b}_{A}(t)\right) \mid t \in A\right\}
$$

where $\mathfrak{a}_{A}(t), \mathfrak{b}_{A}(t): A \longrightarrow[0,1]$ represent the membership grade and nonmembership functions, respectively, with $0 \leq \mathfrak{a}_{A}(t)^{2}+\mathfrak{b}_{A}(t)^{2} \leq 1$, degree of independency $\mathfrak{I}=1-\mathfrak{a}_{A}(t)^{2}-\mathfrak{b}_{A}(t)^{2}$, and $A \subset \mathbb{N}$.

For the sake of readers convenience, we express the PFSN as $\mathscr{H}_{i j}=\left\langle\mathfrak{a}_{i j}, \mathfrak{b}_{i j}\right\rangle$. For calculating the ranking of alternatives, Zulqarnain et al. [30] introduced the score and accuracy functions for $\mathscr{H}_{i j}$ as follows:

$$
S\left(H_{i j}\right)=\mathfrak{a}_{i j}^{2}-\mathfrak{b}_{i j}^{2}
$$

where $S\left(H_{i j}\right) \in[-1,1]$. It is informed that the score function is unable to differentiate the PFSNs in some cases. For example, let $H_{11}=\langle 0.3162,0.4472\rangle$ and $H_{12}=\langle 0.5477$, $0.6324\rangle$, then according to the definition of score function, we have $S\left(H_{11}\right)=-0.1$ and $S\left(H_{12}\right)=-0.1$. So, it is impossible to find the most acceptable alternative utilizing the score function in this case. To handle this drawback, an accuracy function has been developed.

$$
A\left(H_{i j}\right)=\mathfrak{a}_{i j}^{2}+\mathfrak{b}_{i j}^{2},
$$

where $A\left(\mathscr{H}_{i j}\right) \in[-1,1]$.

Thus, to compare two PFSNs $\mathscr{H}_{i j}$ and $\mathscr{R}_{i j}$, following comparison laws are defined:

(1) If $S\left(H_{i j}\right)>S\left(R_{i j}\right)$, then $H_{i j}>H_{i j}$

(2) If $S\left(H_{i j}\right)=S\left(R_{i j}\right)$, then 
(a) If $A\left(H_{i j}\right)>A\left(R_{i j}\right)$, then $H_{i j}>R_{i j}$

(b) If $A\left(H_{i j}\right)=A\left(R_{i j}\right)$, then $H_{i j}=R_{i j}$

\section{Pythagorean Fuzzy Soft Einstein Ordered Weighted Average Operator}

The following section will develop the Einstein ordered weighted average operator for PFSS with some fundamental properties.

Definition 6. Let $\mathrm{H}_{i j}=\left(\mathfrak{a}_{i j}, \mathfrak{b}_{i j}\right)$ be a collection of PFSNs, where $(i=1,2, \ldots, n)$ and $(j=1,2, \ldots m)$, then the Pythagorean fuzzy soft Einstein ordered weighted averaging (PFSEOWA) operator is defined as follows:

$$
\operatorname{PFSEOWA}\left(H_{11}, H_{12}, \ldots, H_{n m}\right)=\oplus_{j=1}^{m} \mathscr{J}_{j}\left(\oplus_{i=1}^{n} \mathcal{O}_{i} H_{\mathfrak{r}(i) \mathfrak{g}(j)}\right) \text {, }
$$

where $\mathscr{O}_{i}$ and $\mathscr{F}_{j}$ represent the weight vectors such that $\mathcal{O}_{i}>0, \sum_{i=1}^{n} \mathcal{O}_{i}=1$, and $\mathscr{J}_{j}>0, \sum_{j=1}^{n} \mathscr{J}_{j}=1$, and $\mathfrak{r}$ and $\mathfrak{G}$ are permutations of $(i=1,2, \ldots n)$ and $(j=1,2, \ldots . m)$ such that $H_{\mathfrak{r}(i-1) j} \geq H_{\mathfrak{r}(i) j}$ and $H_{i \mathfrak{s}(j-1)} \geq H_{i \mathfrak{s}(j)} \forall i, j$.

Theorem 1. Let $H_{i j}=\left(\mathfrak{a}_{i j}, \mathfrak{b}_{i j}\right)$ be a collection of PFSNs, where $(i=1,2, \ldots, n)$ and $(j=1,2, \ldots, m)$, then the aggregated value obtained by equation (9) is given as

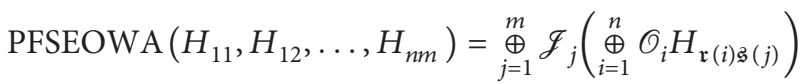

$$
\begin{aligned}
& =\left\langle\frac{\sqrt{\prod_{j=1}^{m}\left(\prod_{i=1}^{n}\left(1+\mathfrak{a}_{\mathfrak{r}(i) \mathfrak{s}(j)}^{2}\right)^{\mathscr{G}_{i}}\right)^{\mathcal{F}_{j}}-\prod_{j=1}^{m}\left(\prod_{i=1}^{n}\left(1-\mathfrak{a}_{\mathfrak{r}(i) \mathfrak{s}(j)}^{2}\right)^{\mathscr{G}_{i}}\right)^{\mathcal{F}_{j}}}}{\sqrt{\prod_{j=1}^{m}\left(\prod_{i=1}^{n}\left(1+\mathfrak{a}_{\mathfrak{r}(i) \mathfrak{s}(j)}^{2}\right)^{\mathscr{G}_{i}}\right)^{\mathcal{F}_{j}}+\prod_{j=1}^{m}\left(\prod_{i=1}^{n}\left(1-\mathfrak{a}_{\mathfrak{r}(i) \mathfrak{s}(j)}^{2}\right)^{\mathscr{G}_{i}}\right)^{\mathcal{F}_{j}}}},\right. \\
& \left.\frac{\sqrt{2 \prod_{j=1}^{m}\left(\prod_{i=1}^{n}\left(\mathfrak{b}_{\mathfrak{r}(i) \mathfrak{s}(j)}^{2}\right)^{\mathcal{G}_{i}}\right)^{\mathcal{F}_{j}}}}{\sqrt{\prod_{j=1}^{m}\left(\prod_{i=1}^{n}\left(2-\mathfrak{b}_{\mathfrak{r}(i) \mathfrak{s}(j)}^{2}\right)^{\mathcal{G}_{i}}\right)^{\mathcal{F}_{j}}+\prod_{j=1}^{m}\left(\prod_{i=1}^{n}\left(\mathfrak{b}_{\mathfrak{r}(i) \mathfrak{s}(j)}^{2}\right)^{\mathcal{G}_{i}}\right)^{\mathcal{F}_{j}}}}\right\rangle,
\end{aligned}
$$

where $\mathscr{O}_{i}$ and $\mathscr{F}_{j}$ represent the weight vectors such that $\mathscr{O}_{i}>0$, $\sum_{i=1}^{n} \mathcal{O}_{i}=1$, and $\mathscr{J}_{j}>0, \sum_{j=1}^{n} \mathscr{F}_{j}=1$, and $\mathfrak{r}$ and $\mathfrak{s}$ are permutations of $(i=1,2, \ldots n)$ and $(j=1,2, \ldots, m)$ such that $H_{\mathfrak{r}(i-1) j} \geq H_{\mathfrak{r}(i) j}$ and $H_{i \mathfrak{s}(j-1)} \geq H_{i \mathfrak{s}(j)} \forall i, j$.
Proof. We will prove it by using mathematical induction. For $n=1$, we get $\mathcal{O}_{i}=1$.

$$
\begin{aligned}
& \operatorname{PFSEOWA}\left(H_{11}, H_{12}, \ldots, H_{n m}\right)=\underset{j=1}{\oplus} \mathscr{J}_{j} H_{\mathfrak{r}(1) \mathfrak{\mathfrak { s }}(j)} \\
& =\left\langle\frac{\sqrt{\prod_{j=1}^{m}\left(1+\mathfrak{a}_{\mathfrak{r}(1) \mathfrak{g}(j)}^{2}\right)^{\mathcal{F}_{j}}-\prod_{j=1}^{m}\left(1-\mathfrak{a}_{\mathfrak{r}(1) \mathfrak{g}(j)}^{2}\right)^{\mathcal{F}_{j}}}}{\sqrt{\prod_{j=1}^{m}\left(1+\mathfrak{a}_{\mathfrak{r}(1) \mathfrak{s}(j)}^{2}\right)^{\mathcal{F}_{j}}+\prod_{j=1}^{m}\left(1-\mathfrak{a}_{\mathfrak{r}(1) \mathfrak{s}(j)}^{2}\right)^{\mathcal{F}_{j}}}},\right. \\
& \left.\frac{\sqrt{2 \prod_{j=1}^{m}\left(\mathfrak{b}_{\mathfrak{r}(1) \mathfrak{s}(j)}^{2}\right)^{\mathscr{F}_{j}}}}{\sqrt{\prod_{j=1}^{m}\left(2-\mathfrak{b}_{\mathfrak{r}(1) \mathfrak{s}(j)}^{2}\right)^{\mathcal{F}_{j}}+\prod_{j=1}^{m}\left(\mathfrak{b}_{\mathfrak{r}(1) \mathfrak{g}(j)}^{2}\right)^{\mathscr{F}_{j}}}}\right\rangle \\
& =\left\langle\frac{\sqrt{\prod_{j=1}^{m}\left(\prod_{i=1}^{1}\left(1+\mathfrak{a}_{\mathfrak{r}(i) \mathfrak{s}(j)}^{2}\right)^{\mathscr{G}_{i}}\right)^{\mathscr{F}_{j}}-\prod_{j=1}^{m}\left(\prod_{i=1}^{1}\left(1-\mathfrak{a}_{\mathfrak{r}(i) \mathfrak{s}(j)}^{2}\right)^{\mathscr{G}_{i}}\right)^{\mathcal{F}_{j}}}}{\sqrt{\prod_{j=1}^{m}\left(\prod_{i=1}^{1}\left(1+\mathfrak{a}_{\mathfrak{r}(i) \mathfrak{g}(j)}^{2}\right)^{\mathscr{G}_{i}}\right)^{\mathcal{F}_{j}}+\prod_{j=1}^{m}\left(\prod_{i=1}^{1}\left(1-\mathfrak{a}_{\mathfrak{r}(i) \mathfrak{s}(j)}^{2}\right)^{\mathcal{G}_{i}}\right)^{\mathscr{F}_{j}}}},\right. \\
& \left.\frac{\sqrt{2 \prod_{j=1}^{m}\left(\prod_{i=1}^{1}\left(\mathfrak{b}_{\mathfrak{r}(i) \mathfrak{s}(j)}^{2}\right)^{\mathscr{G}_{i}}\right)^{\mathcal{F}_{j}}}}{\sqrt{\prod_{j=1}^{m}\left(\prod_{i=1}^{1}\left(2-\mathfrak{b}_{\mathfrak{r}(i) \mathfrak{s}(j)}^{2}\right)^{\mathscr{G}_{i}}\right)^{\mathcal{F}_{j}}+\prod_{j=1}^{m}\left(\prod_{i=1}^{1}\left(\mathfrak{b}_{\mathfrak{r}(i) \mathfrak{s}(j)}^{2}\right)^{\mathscr{G}_{i}}\right)^{\mathcal{F}_{j}}}}\right\rangle .
\end{aligned}
$$

For $m=1$, we get $\mathscr{F}_{j}=1$. 


$$
\begin{aligned}
& \operatorname{PFSEOWA}\left(H_{11}, H_{12}, \ldots, H_{n m}\right)=\bigoplus_{i=1}^{n} \mathcal{O}_{i} H_{\mathfrak{r}(i) \mathfrak{Z}(1)} \\
& =\left\langle\frac{\sqrt{\prod_{i=1}^{n}\left(1+\mathfrak{a}_{\mathfrak{r}(i) \mathfrak{B}(1)}^{2}\right)^{\mathcal{G}_{i}}-\prod_{i=1}^{n}\left(1-\mathfrak{a}_{\mathfrak{r}(i) \mathfrak{g}(1)}^{2}\right)^{\mathcal{G}_{i}}}}{\sqrt{\prod_{i=1}^{n}\left(1+\mathfrak{a}_{\mathfrak{r}(i) \mathfrak{Z}(1)}^{2}\right)^{\mathcal{G}_{i}}+\prod_{i=1}^{n}\left(1-\mathfrak{a}_{\mathfrak{r}(i) \mathfrak{Z}(1)}^{2}\right)^{\mathcal{G}_{i}}}},\right. \\
& \left.\frac{\sqrt{2 \prod_{i=1}^{n}\left(\mathfrak{b}_{\mathfrak{r}(i) \mathfrak{s}(1)}^{2}\right)^{\mathcal{G}_{i}}}}{\sqrt{\prod_{i=1}^{n}\left(2-\mathfrak{b}_{\mathfrak{r}(i) \mathfrak{S}(j)}^{2}\right)^{\mathcal{G}_{i}}+\prod_{i=1}^{n}\left(\mathfrak{b}_{\mathfrak{r}(i) \mathfrak{s}(1)}^{2}\right)^{\mathcal{G}_{i}}}}\right\rangle \\
& =\left\langle\frac{\sqrt{\prod_{j=1}^{1}\left(\prod_{i=1}^{n}\left(1+\mathfrak{a}_{\mathfrak{r}(i) \mathfrak{S}(j)}^{2}\right)^{\mathcal{G}_{i}}\right)^{\mathcal{F}_{j}}-\prod_{j=1}^{1}\left(\prod_{i=1}^{n}\left(1-\mathfrak{a}_{\mathfrak{r}(i) \mathfrak{I}(j)}^{2}\right)^{\mathcal{G}_{i}}\right)^{\mathcal{F}_{j}}}}{\sqrt{\prod_{j=1}^{1}\left(\prod_{i=1}^{n}\left(1+\mathfrak{a}_{\mathfrak{r}(i) \mathfrak{S}(j)}^{2}\right)^{\mathcal{O}_{i}}\right)^{\mathcal{F}_{j}}+\prod_{j=1}^{1}\left(\prod_{i=1}^{n}\left(1-\mathfrak{a}_{\mathfrak{r}(i) \mathfrak{S}(j)}^{2}\right)^{\mathcal{O}_{i}}\right)^{\mathcal{F}_{j}}}}\right. \\
& \left.\frac{\sqrt{2 \prod_{j=1}^{1}\left(\prod_{i=1}^{n}\left(\mathfrak{b}_{\mathfrak{r}(i) \mathfrak{s}(j)}^{2}\right)^{\mathcal{G}_{i}}\right)^{\mathcal{F}_{j}}}}{\sqrt{\prod_{j=1}^{1}\left(\prod_{i=1}^{n}\left(2-\mathfrak{b}_{\mathfrak{r}(i) \mathfrak{z}(j)}^{2}\right)^{\mathcal{G}_{i}}\right)^{\mathcal{F}_{j}}+\prod_{j=1}^{1}\left(\prod_{i=1}^{n}\left(\mathfrak{b}_{\mathfrak{r}(i) \mathfrak{z}(j)}^{2}\right)^{\mathcal{G}_{i}}\right)^{\mathcal{F}_{j}}}}\right\rangle .
\end{aligned}
$$

So, equation (9) is true for $n=1$ and $m=1$.
Suppose that equation holds for $n=\delta_{2}, m=\delta_{1}+1$, and for $n=\delta_{2}+1, m=\delta_{1}$.

$$
\begin{aligned}
& \underset{j=1}{\delta_{1}+1} \mathscr{J}_{j}\left(\underset{i=1}{\oint_{2}} \mathcal{O}_{i} H_{\mathfrak{r}(i) \mathfrak{Z}(j)}\right) \\
& =\left\langle\frac{\sqrt{\prod_{j=1}^{\delta_{1}+1}\left(\prod_{i=1}^{\delta_{2}}\left(1+\mathfrak{a}_{\mathfrak{r}(i) \mathfrak{b}(j)}^{2}\right)^{\mathcal{O}_{i}}\right)^{\mathcal{F}_{j}}-\prod_{j=1}^{\delta_{1}+1}\left(\prod_{i=1}^{\delta_{2}}\left(1-\mathfrak{a}_{\mathfrak{r}(i) \mathfrak{z}(j)}^{2}\right)^{\mathcal{G}_{i}}\right)^{\mathcal{F}_{j}}}}{\sqrt{\prod_{j=1}^{\delta_{1}+1}\left(\prod_{i=1}^{\delta_{2}}\left(1+\mathfrak{a}_{\mathfrak{r}(i) \mathfrak{z}(j)}^{2}\right)^{\mathcal{G}_{i}}\right)^{\mathcal{F}_{j}}+\prod_{j=1}^{\delta_{1}+1}\left(\prod_{i=1}^{\delta_{2}}\left(1-\mathfrak{a}_{\mathfrak{r}(i) \mathfrak{l}(j)}^{2}\right)^{\mathcal{G}_{i}}\right)^{\mathcal{F}_{j}}}},\right. \\
& \left.\frac{\sqrt{2 \prod_{j=1}^{\delta_{1}+1}\left(\prod_{i=1}^{\delta_{2}}\left(\mathfrak{b}_{\mathfrak{r}(i) \mathfrak{s}(j)}^{2}\right)^{\mathcal{G}_{i}}\right)^{\mathcal{F}_{j}}}}{\sqrt{\prod_{j=1}^{\delta_{1}+1}\left(\prod_{i=1}^{\delta_{2}}\left(2-\mathfrak{b}_{\mathfrak{r}(i) \mathfrak{I}(j)}^{2}\right)^{\mathcal{G}_{i}}\right)^{\mathcal{F}_{j}}+\prod_{j=1}^{\delta_{1}+1}\left(\prod_{i=1}^{\delta_{2}}\left(\mathfrak{b}_{\mathfrak{r}(i) \mathfrak{S}(j)}^{2}\right)^{\mathcal{O}_{i}}\right)^{\mathcal{F}_{j}}}}\right\rangle,
\end{aligned}
$$

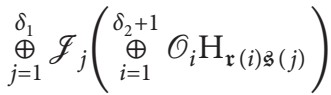

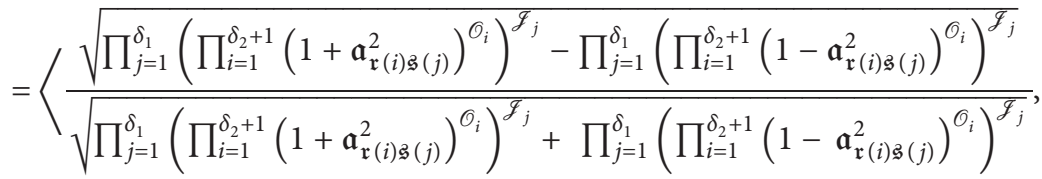

$$
\begin{aligned}
& \left.\frac{\sqrt{2 \prod_{j=1}^{\delta_{1}}\left(\prod_{i=1}^{\delta_{2}+1}\left(\mathfrak{b}_{\mathfrak{r}(i) \mathfrak{g}(j)}^{2}\right)^{\mathcal{G}_{i}}\right)^{\mathcal{F}_{j}}}}{\sqrt{\prod_{j=1}^{\delta_{1}}\left(\prod_{i=1}^{\delta_{2}+1}\left(2-\mathfrak{b}_{\mathfrak{r}(i) \mathfrak{I}(j)}^{2}\right)^{\mathcal{G}_{i}}\right)^{\mathcal{F}_{j}}+\prod_{j=1}^{\delta_{1}}\left(\prod_{i=1}^{\delta_{2}+1}\left(\mathfrak{b}_{\mathfrak{r}(i) \mathfrak{z}(j)}^{2}\right)^{\mathcal{G}_{i}}\right)^{\mathcal{F}_{j}}}}\right\rangle .
\end{aligned}
$$

Now, we prove the equation for $m=\delta_{1}+1$ and $n=\delta_{2}+1$ : 


$$
\begin{aligned}
& \underset{j=1}{\delta_{1}+1} \mathscr{J}_{j}\left(\oplus_{i=1}^{\delta_{2}+1} \mathcal{O}_{i} H_{\mathfrak{r}(i) \mathfrak{I}(j)}\right)=\underset{j=1}{\delta_{1}+1} H_{\mathfrak{Z}(j)}\left(\underset{i=1}{\oint_{2}} \mathcal{O}_{i} H_{\mathfrak{r}(i) \mathfrak{z}(j)} \oplus \mathcal{O}_{i+1} H_{\mathfrak{r}\left(\delta_{2}+1\right) \mathfrak{I}(j)}\right)
\end{aligned}
$$

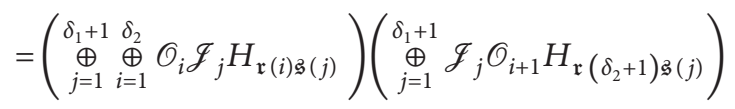

$$
\begin{aligned}
& =\left\langle\frac{\sqrt{2 \prod_{j=1}^{\delta_{1}+1}\left(\prod_{i=1}^{\delta_{2}}\left(\mathfrak{b}_{\mathfrak{r}(i) \mathfrak{I}(j)}^{2}\right)^{\mathcal{G}_{i}}\right)^{\mathcal{F}_{j}}}}{\sqrt{\prod_{j=1}^{\delta_{1}+1}\left(\prod_{i=1}^{\delta_{2}}\left(2-\mathfrak{b}_{\mathfrak{r}(i) \mathfrak{I}(j)}^{2}\right)^{\mathcal{G}_{i}}\right)^{\mathcal{F}_{j}}+\prod_{j=1}^{\delta_{1}+1}\left(\prod_{i=1}^{\delta_{2}}\left(\mathfrak{b}_{\mathfrak{r}(i) \mathfrak{I}(j)}^{2}\right)^{\mathcal{G}_{i}}\right)^{\mathcal{F}_{j}}}}\right. \\
& \left.\oplus \frac{\sqrt{2 \prod_{j=1}^{\delta_{1}+1}\left(\left(\mathfrak{b}_{\mathfrak{r}\left(\delta_{2}+1\right) \mathfrak{s}(j)}^{2}\right)^{\mathcal{O}_{\delta_{2}+1}}\right)^{\mathcal{F}_{j}}}}{\sqrt{\prod_{j=1}^{\delta_{1}+1}\left(\left(2-\mathfrak{b}_{\mathfrak{r}\left(\delta_{2}+1\right) \mathfrak{z}(j)}^{2}\right)^{\mathcal{O}_{\delta_{2}+1}}\right)^{\mathcal{F}_{j}}+\prod_{j=1}^{\delta_{1}+1}\left(\left(\mathfrak{b}_{\mathfrak{r}\left(\delta_{2}+1\right) \mathfrak{z}(j)}^{2}\right)^{\mathcal{O}_{\delta_{2}+1}}\right)^{\mathcal{F}_{j}}}}\right\rangle \\
& =\left\langle\frac{\sqrt{\prod_{j=1}^{\delta_{1}+1}\left(\prod_{i=1}^{\delta_{2}+1}\left(1+\mathfrak{a}_{\mathfrak{r}(i) \mathfrak{B}(j)}^{2}\right)^{\mathcal{G}_{i}}\right)^{\mathcal{F}_{j}}-\prod_{j=1}^{\delta_{1}+1}\left(\prod_{i=1}^{\delta_{2}+1}\left(1-\mathfrak{a}_{\mathfrak{r}(i) \mathfrak{s}(j)}^{2}\right)^{\mathcal{G}_{i}}\right)^{\mathcal{F}_{j}}}}{\sqrt{\prod_{j=1}^{\delta_{1}+1}\left(\prod_{i=1}^{\delta_{2}+1}\left(1+\mathfrak{a}_{\mathfrak{r}(i) \mathfrak{I}(j)}^{2}\right)^{\mathcal{G}_{i}}\right)^{\mathcal{J}_{j}}+\prod_{j=1}^{\delta_{1}+1}\left(\prod_{i=1}^{\delta_{2}+1}\left(1-\mathfrak{a}_{\mathfrak{r}(i) \mathfrak{I}(j)}^{2}\right)^{\mathcal{O}_{i}}\right)^{\mathcal{J}_{j}}}}\right. \\
& \left.\frac{\sqrt{2 \prod_{j=1}^{\delta_{1}+1}\left(\prod_{i=1}^{\delta_{2}+1}\left(\mathfrak{b}_{\mathfrak{r}(i) \mathfrak{s}(j)}^{2}\right)^{\mathcal{G}_{i}}\right)^{\mathcal{F}_{j}}}}{\sqrt{\prod_{j=1}^{\delta_{1}+1}\left(\prod_{i=1}^{\delta_{2}+1}\left(2-\mathfrak{b}_{\mathfrak{r}(i) \mathfrak{S}(j)}^{2}\right)^{\mathcal{G}_{i}}\right)^{\mathcal{F}_{j}}+\prod_{j=1}^{\delta_{1}+1}\left(\prod_{i=1}^{\delta_{2}+1}\left(\mathfrak{b}_{\mathfrak{r}(i) \mathfrak{g}(j)}^{2}\right)^{\mathcal{G}_{i}}\right)^{\mathcal{F}_{j}}}}\right\rangle \\
& =\bigoplus_{j=1}^{\delta_{1}+1} \mathscr{J}_{j}\left(\underset{i=1}{\delta_{2}+1} \mathcal{O}_{i} \mathrm{H}_{\mathfrak{r}(i) \mathfrak{I}(j)}\right)
\end{aligned}
$$

So, it is valid for $m=\delta_{1}+1$ and $n=\delta_{2}+1$.

Example 1. Let $R=\left\{R_{1}, R_{2}, R_{3}\right\}$ be a set of decision-makers with weight vector $\mathscr{O}_{i}=(0.1,0.3,0.3,0.3)^{T}$, who want to decide a bike under the set of attributes $A=\left\{A_{1}=\right.$ fuel milage, $A_{2}=$ speed per hour, $\quad A_{3}=$ price, $A_{4}=$ comfort level, $A_{5}=$ design $\}$ with weight vector $\mathscr{J}_{j}=(0.2$, $0.2,0.2,0.4)^{T}$. The assumed rating values for each attribute in the form of PFSNs $\left(\mathrm{H}_{4 \times 4}, A\right)=\left(\mathfrak{a}_{i j}, \mathfrak{b}_{i j}\right)_{4 \times 4}$ are given as follows:

$$
\left(\mathrm{H}_{4 \times 4}, A\right)=\left[\begin{array}{cccc}
(0.5,0.8) & (0.7,0.5) & (0.4,0.6) & (0.7,0.4) \\
(0.5,0.6) & (0.9,0.1) & (0.3,0.7) & (0.4,0.5) \\
(0.4,0.8) & (0.7,0.5) & (0.4,0.6) & (0.3,0.5) \\
(0.3,0.7) & (0.6,0.5) & (0.5,0.4) & (0.5,0.7)
\end{array}\right]
$$


First, we find the associated ordered position matrix by using the score function, which is as follows:

$$
\left(\mathrm{H}_{4 \times 4}, A\right)=\left[\begin{array}{cccc}
(0.7,0.4) & (0.7,0.5) & (0.4,0.6) & (0.5,0.8) \\
(0.9,0.1) & (0.4,0.5) & (0.3,0.7) & (0.5,0.6) \\
(0.7,0.5) & (0.4,0.6) & (0.3,0.5) & (0.4,0.8) \\
(0.6,0.5) & (0.5,0.4) & (0.3,0.7) & (0.5,0.7)
\end{array}\right]
$$

As we know,

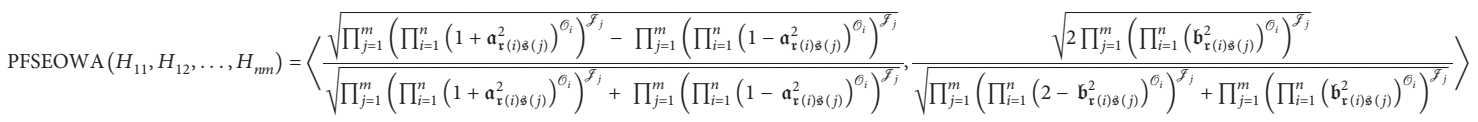

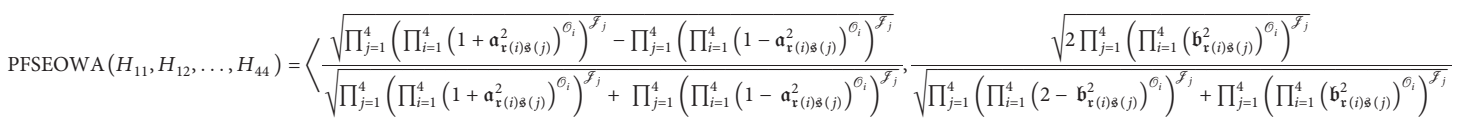

$$
\begin{aligned}
& \left.\frac{\sqrt{2\left[\left\{(0.16)^{0.1}(0.01)^{0.3}(0.25)^{0.3}(0.25)^{0.3}\right\}^{0.2}\left\{(0.25)^{0.1}(0.25)^{0.3}(0.36)^{0.3}(0.16)^{0.3}\right\}^{0.2}\left\{(0.36)^{0.1}(0.49)^{0.3}(0.25)^{0.3}(0.49)^{0.3}\right\}^{0.2}\left\{(0.64)^{0.1}(0.36)^{0.3}(0.64)^{0.3}(0.49)^{0.3}\right\}^{0.4}\right]}}{\sqrt{\left\{(1.84)^{0.1}(1.99)^{0.3}(1.75)^{0.3}(1.75)^{0.3}\right\}^{0.2}\left\{(1.75)^{0.1}(1.75)^{0.3}(1.64)^{0.3}(1.84)^{0.3}\right\}^{0.2}\left\{(1.64)^{0.1}(1.51)^{0.3}(1.36)^{0.3}(1.51)^{0.3}\right\}^{0.2}\left\{(1.36)^{0.1}(1.64)^{0.3}(1.36)^{0.3}(1.51)^{0.3}\right\}^{0.4}}+}\right\rangle \\
& \sqrt{\left\{(0.16)^{0.1}(0.01)^{0.3}(0.25)^{0.3}(0.25)^{0.3}\right\}^{0.2}\left\{(0.25)^{0.1}(0.25)^{0.3}(0.36)^{0.3}(0.16)^{0.3}\right\}^{0.2}\left\{(0.36)^{0.1}(0.49)^{0.3}(0.25)^{0.3}(0.49)^{0.3}\right\}^{0.2}\left\{(0.64)^{0.1}(0.36)^{0.3}(0.64)^{0.3}(0.49)^{0.3}\right\}^{0.4}} \\
& =\langle 0.5283,0.5242\rangle \text {. }
\end{aligned}
$$

$$
\operatorname{PFSEOWA}\left(H_{11}, H_{12}, \ldots, H_{n m}\right)=H,
$$

\subsection{Properties of PFSEOWA Operator}

3.1.1. Idempotency. Let $H_{i j}=\left(\mathfrak{a}_{i j}, \mathfrak{b}_{i j}\right)$ be a collection of PFSNs, where $(i=1,2, \ldots, n)$ and $(j=1,2, \ldots, m)$.

If $H_{\mathfrak{r}(i) \mathfrak{g}(j)}=H_{11}$ are mathematically identical, then where $\mathscr{O}_{i}$ and $\mathscr{F}_{j}$ represent the weight vectors such that $\mathcal{O}_{i}>0, \sum_{i=1}^{n} \mathcal{O}_{i}=1$, and $\mathscr{J}_{j}>0, \sum_{j=1}^{n} \mathscr{J}_{j}=1$.

Proof. As we know, 
$\operatorname{PFSEOWA}\left(H_{11}, H_{12}, \ldots, H_{n m}\right)$

$$
\begin{aligned}
& =\left\langle\frac{\sqrt{\prod_{j=1}^{m}\left(\prod_{i=1}^{n}\left(1+\mathfrak{a}_{\mathfrak{r}(i) \mathfrak{s}(j)}^{2}\right)^{\mathscr{G}_{i}}\right)^{\mathscr{F}_{j}}-\prod_{j=1}^{m}\left(\prod_{i=1}^{n}\left(1-\mathfrak{a}_{\mathfrak{r}(i) \mathfrak{s}(j)}^{2}\right)^{\mathcal{G}_{i}}\right)^{\mathscr{F}_{j}}}}{\sqrt{\prod_{j=1}^{m}\left(\prod_{i=1}^{n}\left(1+\mathfrak{a}_{\mathfrak{r}(i) \mathfrak{s}(j)}^{2}\right)^{\mathscr{G}_{i}}\right)^{\mathscr{F}_{j}}+\prod_{j=1}^{m}\left(\prod_{i=1}^{n}\left(1-\mathfrak{a}_{\mathfrak{r}(i) \mathfrak{s}(j)}^{2}\right)^{\mathscr{G}_{i}}\right)^{\mathscr{F}_{j}}}},\right. \\
& \left.\frac{\sqrt{2 \prod_{j=1}^{m}\left(\prod_{i=1}^{n}\left(\mathfrak{b}_{\mathfrak{r}(i) \mathfrak{s}(j)}^{2}\right)^{\mathcal{G}_{i}}\right)^{\mathcal{F}_{j}}}}{\sqrt{\prod_{j=1}^{m}\left(\prod_{i=1}^{n}\left(2-\mathfrak{b}_{\mathfrak{r}(i) \mathfrak{g}(j)}^{2}\right)^{\mathcal{G}_{i}}\right)^{\mathcal{F}_{j}}+\prod_{j=1}^{m}\left(\prod_{i=1}^{n}\left(\mathfrak{b}_{\mathfrak{r}(i) \mathfrak{g}(j)}^{2}\right)^{\mathcal{G}_{i}}\right)^{\mathcal{F}_{j}}}}\right\rangle
\end{aligned}
$$

As $H_{\mathfrak{r}(i) \mathfrak{z}(j)}=H_{i j}$, So

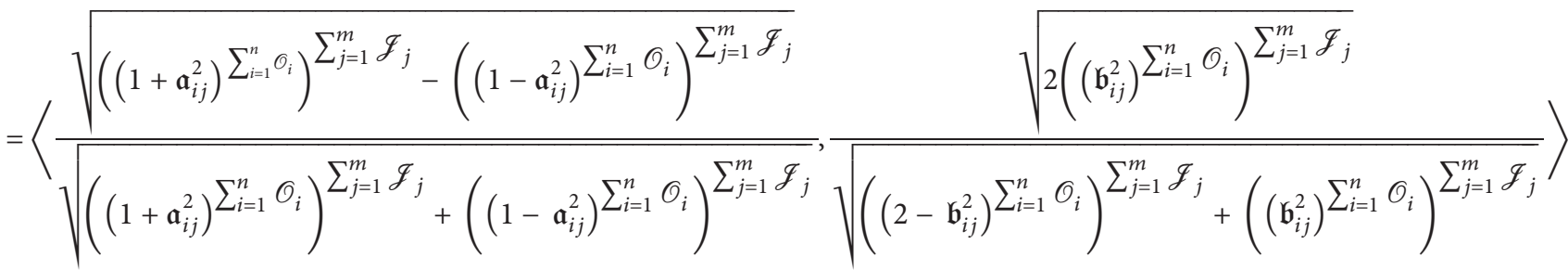

$$
\begin{aligned}
& =\left\langle\frac{\sqrt{\left(1+\mathfrak{a}_{i j}^{2}\right)-\left(1-\mathfrak{a}_{i j}^{2}\right)}}{\sqrt{\left(1+\mathfrak{a}_{i j}^{2}\right)+\left(1-\mathfrak{a}_{i j}^{2}\right)}}, \frac{\sqrt{2 \mathfrak{b}_{i j}^{2}}}{\sqrt{\left(2-\mathfrak{b}_{i j}^{2}\right)+\left(\mathfrak{b}_{i j}^{2}\right)}}\right\rangle \\
& =\left\langle\mathfrak{a}_{i j}, \mathfrak{b}_{i j}\right\rangle=H \text {. }
\end{aligned}
$$

3.1.2. Boundedness. Let $H_{i j}=\left(\mathfrak{a}_{i j}, \mathfrak{b}_{i j}\right)$ be a collection of PFSNs, where $(i=1,2, \ldots, n)$ and $(j=1,2, \ldots, m)$. then

If $H_{\min }=\min \left(H_{\mathbf{r}(i) \mathfrak{g}(j)}\right)$ and $H_{\max }=\max \left(H_{\mathfrak{r}(i) \mathfrak{g}(j)}\right)$,

$$
H_{\min } \leq \operatorname{PFSEOWA}\left(H_{11}, H_{12}, \ldots, H_{n m}\right) \leq H_{\max },
$$

where $\mathscr{O}_{i}$ and $\mathscr{F}_{j}$ represent the weight vectors such that $\mathcal{O}_{i}>0, \sum_{i=1}^{n} \mathcal{O}_{i}=1$, and $\mathscr{J}_{j}>0, \sum_{j=1}^{n} \mathscr{J}_{j}=1$.

Proof. Let $\left.\left.g(y)=\sqrt{\left(1-y^{2}\right) /\left(1+y^{2}\right)}, \quad y \in\right] 0,1\right]$, then $(\mathrm{d} / \mathrm{d} y)(g(y))=-2 y /\left(1+y^{2}\right)^{2} \sqrt{\left(1+y^{2}\right) /\left(1-y^{2}\right)}<0$, which shows that $g(y)$ is decreasing function on $] 0,1]$. So, $\mathfrak{a}_{\min } \leq \mathfrak{a}_{\mathfrak{r}(i) \mathfrak{z}(j)} \leq \mathfrak{a}_{\max }$.

Hence, $g\left(\mathfrak{a}_{\max }\right) \leq g\left(\mathfrak{a}_{\mathfrak{r}(i) \mathfrak{s}(j)}\right) \leq g\left(\mathfrak{a}_{\min }\right)$ :

$$
\Rightarrow \sqrt{\frac{1-\mathfrak{a}_{\max }^{2}}{1+\mathfrak{a}_{\max }^{2}}} \leq \sqrt{\frac{1-\mathfrak{a}_{\mathfrak{r}(i) \mathfrak{g}(j)}^{2}}{1+\mathfrak{a}_{\mathfrak{r}(i) \mathfrak{g}(j)}^{2}}} \leq \sqrt{\frac{1-\mathfrak{a}_{\min }^{2}}{1+\mathfrak{a}_{\min }^{2}}} .
$$

Let $\mathcal{O}_{i}$ and $\mathscr{F}_{j}$ represent the weight vectors such that $\mathcal{O}_{i}>0, \sum_{i=1}^{n} \mathcal{O}_{i}=1$, and $\mathscr{J}_{j}>0, \sum_{j=1}^{n} \mathscr{J}_{j}=1$. Then, we have

$$
\begin{aligned}
& \Longleftrightarrow \sqrt{\prod_{j=1}^{m}\left(\prod_{i=1}^{n}\left(\frac{1-\mathfrak{a}_{\max }^{2}}{1+\mathfrak{a}_{\max }^{2}}\right)^{\mathcal{O}_{i}}\right)^{\mathcal{F}_{j}}} \leq \sqrt{\prod_{j=1}^{m}\left(\prod_{i=1}^{n}\left(\frac{1-\mathfrak{a}_{\mathfrak{r}(i) \mathfrak{g}(j)}^{2}}{1+\mathfrak{a}_{\mathfrak{r}(i) \mathfrak{F}(j)}^{2}}\right)^{\mathcal{G}_{i}}\right)^{\mathcal{F}_{j}}} \leq \sqrt{\prod_{j=1}^{m}\left(\prod_{i=1}^{n}\left(\frac{1-\mathfrak{a}_{\min }^{2}}{1+\mathfrak{a}_{\min }^{2}}\right)^{\mathscr{G}_{i}}\right)^{\mathcal{F}_{j}}} \\
& \Longleftrightarrow \sqrt{\left(\left(\frac{1-\mathfrak{a}_{\max }^{2}}{1+\mathfrak{a}_{\max }^{2}}\right)^{\sum_{i=1}^{n} \mathscr{O}_{i}}\right)^{\sum_{j=1}^{m} \mathcal{F}_{j}}} \leq \sqrt{\prod_{j=1}^{m}\left(\prod_{i=1}^{n}\left(\frac{1-\mathfrak{a}_{\mathfrak{r}(i) \mathfrak{g}(j)}^{2}}{1+\mathfrak{a}_{\mathfrak{r}(i) \mathfrak{B}(j)}^{2}}\right)^{\mathcal{O}_{i}}\right)^{\mathcal{F}_{j}}} \leq \sqrt{\left(\left(\frac{1-\mathfrak{a}_{\min }^{2}}{1+\mathfrak{a}_{\min }^{2}}\right)^{\sum_{i=1}^{n} \mathscr{O}_{i}}\right)^{\sum_{j=1}^{m} \mathscr{F}_{j}}}
\end{aligned}
$$


Mathematical Problems in Engineering

9

$$
\begin{aligned}
& \Longleftrightarrow \sqrt{1+\left(\frac{1-\mathfrak{a}_{\max }^{2}}{1+\mathfrak{a}_{\max }^{2}}\right)} \leq \sqrt{1+\prod_{j=1}^{m}\left(\prod_{i=1}^{n}\left(\frac{1-\mathfrak{a}_{\mathfrak{r}(i) \mathfrak{I}(j)}^{2}}{1+\mathfrak{a}_{\mathfrak{r}(i) \mathfrak{I}(j)}^{2}}\right)^{\mathcal{G}_{i}}\right)^{\mathcal{F}_{j}}} \leq \sqrt{1+\left(\frac{1-\mathfrak{a}_{\min }^{2}}{1+\mathfrak{a}_{\min }^{2}}\right)} \\
& \Longleftrightarrow \sqrt{\frac{2}{1+\mathfrak{a}_{\max }^{2}}} \leq \sqrt{1+\prod_{j=1}^{m}\left(\prod_{i=1}^{n}\left(\frac{1-\mathfrak{a}_{\mathfrak{r}(i) \mathfrak{z}(j)}^{2}}{1+\mathfrak{a}_{\mathfrak{r}(i) \mathfrak{z}(j)}^{2}}\right)^{\mathcal{O}_{i}}\right)^{\mathcal{F}_{j}}} \leq \sqrt{\frac{2}{1+\mathfrak{a}_{\min }^{2}}} \\
& \Longleftrightarrow \sqrt{\frac{1+\mathfrak{a}_{\min }^{2}}{2}} \leq \frac{1}{\sqrt{1+\prod_{j=1}^{m}\left(\prod_{i=1}^{n}\left(\left(1-\mathfrak{a}_{\mathfrak{r}(i) \mathfrak{b}(j)}^{2}\right) /\left(1+\mathfrak{a}_{\mathfrak{r}(i) \mathfrak{s}(j)}^{2}\right)\right)^{\mathcal{G}_{i}}\right)^{\mathcal{F}_{j}}}} \leq \sqrt{\frac{1+\mathfrak{a}_{\max }^{2}}{2}} \\
& \Longleftrightarrow \sqrt{1+\mathfrak{a}_{\min }^{2}} \leq \sqrt{\frac{2}{1+\prod_{j=1}^{m}\left(\prod_{i=1}^{n}\left(\left(1-\mathfrak{a}_{\mathfrak{r}(i) \mathfrak{s}(j)}^{2}\right) /\left(1+\mathfrak{a}_{\mathfrak{r}(i) \mathfrak{s}(j)}^{2}\right)\right)^{\mathcal{G}_{i}}\right)^{\mathcal{I}}}} \leq \sqrt{1+\mathfrak{a}_{\max }^{2}} \\
& \Longleftrightarrow \sqrt{1+\mathfrak{a}_{\min }^{2}-1} \leq \sqrt{\frac{2}{1+\prod_{j=1}^{m}\left(\prod_{i=1}^{n}\left(\left(1-\mathfrak{a}_{\mathfrak{r}(i) \mathfrak{z}(j)}^{2}\right) /\left(1+\mathfrak{a}_{\mathfrak{r}(i) \mathfrak{g}(j)}^{2}\right)\right)^{\mathcal{G}_{i}}\right)^{\mathcal{F}_{j}}}-1} \leq \sqrt{1+\mathfrak{a}_{\max }^{2}-1} \\
& \Longleftrightarrow \sqrt{\mathfrak{a}_{\min }^{2}} \leq \sqrt{\frac{2}{1+\prod_{j=1}^{m}\left(\prod_{i=1}^{n}\left(\left(1-\mathfrak{a}_{\mathfrak{r}(i) \mathfrak{B}(j)}^{2}\right) /\left(1+\mathfrak{a}_{\mathfrak{r}(i) \mathfrak{z}(j)}^{2}\right)\right)^{\mathcal{G}_{i}}\right)^{\mathcal{F}_{j}}}-1} \leq \sqrt{\mathfrak{a}_{\max }^{2}} \\
& \Longleftrightarrow \mathfrak{a}_{\min } \leq \sqrt{\frac{2}{1+\prod_{j=1}^{m}\left(\prod_{i=1}^{n}\left(\left(1-\mathfrak{a}_{\mathfrak{r}(i) \mathfrak{z}(j)}^{2}\right) /\left(1+\mathfrak{a}_{\mathfrak{r}(i) \mathfrak{z}(j)}^{2}\right)\right)^{\mathcal{G}_{i}}\right)^{\mathcal{F}_{j}}}-1} \leq \mathfrak{a}_{\max } \\
& \mathfrak{a}_{\min } \leq \frac{\sqrt{\prod_{j=1}^{m}\left(\prod_{i=1}^{n}\left(1+\mathfrak{a}_{\mathfrak{r}(i) \mathfrak{g}(j)}^{2}\right)^{\mathcal{G}_{i}}\right)^{\mathcal{F}_{j}}-\prod_{j=1}^{m}\left(\prod_{i=1}^{n}\left(1-\mathfrak{a}_{\mathfrak{r}(i) \mathfrak{g}(j)}^{2}\right)^{\mathcal{G}_{i}}\right)^{\mathcal{F}_{j}}}}{\sqrt{\prod_{j=1}^{m}\left(\prod_{i=1}^{n}\left(1+\mathfrak{a}_{\mathfrak{r}(i) \mathfrak{g}(j)}^{2}\right)^{\mathcal{O}_{i}}\right)^{\mathcal{F}_{j}}+\prod_{j=1}^{m}\left(\prod_{i=1}^{n}\left(1-\mathfrak{a}_{\mathfrak{r}(i) \mathfrak{s}(j)}^{2}\right)^{\mathcal{G}_{i}}\right)^{\mathcal{F}_{j}}}} \leq \mathfrak{a}_{\max } .
\end{aligned}
$$

Let $\left.\left.f(x)=\sqrt{\left(2-x^{2}\right) / x^{2}}, \quad x \in\right] 0,1\right]$, then $(\mathrm{d} / \mathrm{d} x)(f$ $(x))=\left(-2 / x^{3}\right) \sqrt{x^{2} /\left(2-x^{2}\right)}<0$. So, $f(x)$ is decreasing function on $] 0,1]$.

As $\mathfrak{b}_{\text {min }} \leq \mathfrak{b}_{\mathfrak{r}(i) \mathfrak{g}(j)} \leq \mathfrak{b}_{\max }, \forall i, j$, so $f\left(\mathfrak{b}_{\max }\right) \leq f\left(\mathfrak{b}_{\mathfrak{r}(i) \mathfrak{g}(j)}\right)$

$$
\begin{aligned}
& \left.\leq f\left(\mathfrak{b}_{\min }\right) \text { and } \sqrt{\left(2-\mathfrak{b}_{\max }^{2}\right) / \mathfrak{b}_{\max }^{2}} \leq \sqrt{(2-} \mathfrak{b}_{\mathfrak{r}(i) \mathfrak{g}(j)}^{2}\right) / \mathfrak{b}_{\mathfrak{r}(i) \mathfrak{g}(j)}^{2} \\
& \leq \sqrt{\left(2-\mathfrak{b}_{\min }^{2}\right) / \mathfrak{b}_{\text {min }}^{2}} .
\end{aligned}
$$

Let $\mathcal{O}_{i}$ and $\mathscr{J}_{j}$ represent the weight vectors such that $\mathcal{O}_{i}>0, \sum_{i=1}^{n} \mathcal{O}_{i}=1$, and $\mathscr{J}_{j}>0, \sum_{j=1}^{n} \mathscr{J}_{j}=1$, then we have

$$
\begin{aligned}
& \Longleftrightarrow \sqrt{\prod_{j=1}^{m}\left(\prod_{i=1}^{n}\left(\frac{2-\mathfrak{b}_{\max }^{2}}{\mathfrak{b}_{\max }^{2}}\right)^{\mathcal{G}_{i}}\right)^{\mathcal{F}_{j}}} \leq \sqrt{\prod_{j=1}^{m}\left(\prod_{i=1}^{n}\left(\frac{2-\mathfrak{b}_{\mathfrak{r}(i) \mathfrak{g}(j)}^{2}}{\mathfrak{b}_{\mathfrak{r}(i) \mathfrak{g}(j)}^{2}}\right)^{\mathcal{G}_{i}}\right)^{\mathcal{F}_{j}}} \leq \sqrt{\prod_{j=1}^{m}\left(\prod_{i=1}^{n}\left(\frac{2-\mathfrak{b}_{\min }^{2}}{\mathfrak{b}_{\min }^{2}}\right)^{\mathcal{G}_{i}}\right)^{\mathcal{F}_{j}}} \\
& \Longleftrightarrow \sqrt{\left(\left(\frac{2-\mathfrak{b}_{\max }^{2}}{\mathfrak{b}_{\max }^{2}}\right)^{\sum_{i=1}^{n} \mathscr{G}_{i}}\right)^{\sum_{j=1}^{m} \mathscr{F}_{j}}} \leq \sqrt{\prod_{j=1}^{m}\left(\prod_{i=1}^{n}\left(\frac{2-\mathfrak{b}_{\mathfrak{r}(i) \mathfrak{g}(j)}^{2}}{\mathfrak{b}_{\mathfrak{r}(i) \mathfrak{g}(j)}^{2}}\right)^{\mathcal{G}_{i}}\right)^{\mathscr{G} j}} \leq \sqrt{\left(\left(\frac{2-\mathfrak{b}_{\min }^{2}}{\mathfrak{b}_{\min }^{2}}\right)^{\sum_{i=1}^{n} \mathscr{G}_{i}}\right)^{\sum_{j=1}^{m} \mathscr{F}_{j}}}
\end{aligned}
$$




$$
\begin{aligned}
& \Longleftrightarrow \sqrt{1+\left(\frac{2-\mathfrak{b}_{\max }^{2}}{\mathfrak{b}_{\max }^{2}}\right)} \leq \sqrt{1+\prod_{j=1}^{m}\left(\prod_{i=1}^{n}\left(\frac{2-\mathfrak{b}_{\mathfrak{r}(i) \mathfrak{g}(j)}^{2}}{\mathfrak{b}_{\mathfrak{r}(i) \mathfrak{g}(j)}^{2}}\right)^{\mathcal{G}_{i}}\right)^{\mathcal{F}_{j}}} \leq \sqrt{1+\left(\frac{2-\mathfrak{b}_{\min }^{2}}{\mathfrak{b}_{\min }^{2}}\right)} \\
& \Longleftrightarrow \sqrt{\frac{2}{\mathfrak{b}_{\max }^{2}}} \leq \sqrt{1+\prod_{j=1}^{m}\left(\prod_{i=1}^{n}\left(\frac{2-\mathfrak{b}_{\mathfrak{r}(i) \mathfrak{g}(j)}^{2}}{\mathfrak{b}_{\mathfrak{r}(i) \mathfrak{g}(j)}^{2}}\right)^{\mathcal{G}_{i}}\right)^{\mathcal{F}_{j}}} \leq \sqrt{\frac{2}{\mathfrak{b}_{\min }^{2}}} \\
& \Longleftrightarrow \sqrt{\frac{\mathfrak{b}_{\min }^{2}}{2}} \leq \frac{1}{\sqrt{1+\prod_{j=1}^{m}\left(\prod_{i=1}^{n}\left(\left(2-\mathfrak{b}_{\mathfrak{r}(i) \mathfrak{g}(j)}^{2}\right) / \mathfrak{b}_{\mathfrak{r}(i) \mathfrak{z}(j)}^{2}\right)^{\mathcal{G}_{i}}\right)^{\mathcal{F}_{j}}}} \leq \sqrt{\frac{\mathfrak{b}_{\text {max }}^{2}}{2}} \\
& \Longleftrightarrow \mathfrak{b}_{\min } \leq \sqrt{\frac{2}{1+\prod_{j=1}^{m}\left(\prod_{i=1}^{n}\left(\left(2-\mathfrak{b}_{\mathfrak{r}(i) \mathfrak{z}(j)}^{2}\right) / \mathfrak{b}_{\mathfrak{r}(i) \mathfrak{g}(j)}^{2}\right)^{\mathcal{O}_{i}}\right)^{\mathcal{F}_{j}}}} \leq \mathfrak{b}_{\max } \\
& \mathfrak{b}_{\min } \leq \frac{\sqrt{2 \prod_{j=1}^{m}\left(\prod_{i=1}^{n}\left(\mathfrak{b}_{\mathfrak{r}(i) \mathfrak{g}(j)}^{2}\right)^{\mathcal{G}_{i}}\right)^{\mathcal{F}_{j}}}}{\sqrt{\prod_{j=1}^{m}\left(\prod_{i=1}^{n}\left(2-\mathfrak{b}_{\mathfrak{r}(i) \mathfrak{g}(j)}^{2}\right)^{\mathcal{G}_{i}}\right)^{\mathcal{F}_{j}}+\prod_{j=1}^{m}\left(\prod_{i=1}^{n}\left(\mathfrak{b}_{\mathfrak{r}(i) \mathfrak{I}(j)}^{2}\right)^{\mathcal{G}_{i}}\right)^{\mathcal{F}_{j}}}} \leq \mathfrak{b}_{\max } .
\end{aligned}
$$

Let

$$
\operatorname{PFSEOWA}\left(H_{11}, H_{12}, \ldots, H_{n m}\right)=\mathscr{H}
$$

Then, equations (22) and (23) can be written as $\mathfrak{a}_{\min } \leq \mathfrak{a} \leq \mathfrak{a}_{\max }$ and $\mathfrak{b}_{\min } \leq \mathfrak{b} \leq \mathfrak{b}_{\max }$. $S(H)=\mathfrak{a}^{2}-\mathfrak{b}^{2} \leq \mathfrak{a}_{\max }^{2}-\mathfrak{b}_{\min }^{2}=S\left(H_{\max }\right)$ and

$$
S(H)=\mathfrak{a}^{2}-\mathfrak{b}^{2} \geq \mathfrak{a}_{\min }^{2}-\mathfrak{b}_{\max }^{2}=S\left(H_{\min }\right) .
$$

If $S(H)<S\left(H_{\max }\right)$ and $S(H)>S\left(H_{\min }\right)$, then we have

$$
H_{\min }<\operatorname{PFSEOWA}\left(H_{11}, H_{12}, \ldots, H_{m}\right)<H_{\max } \cdot
$$

If $S(H)=S\left(H_{\max }\right)$, then we have $\mathfrak{a}^{2}=\mathfrak{a}_{\max }^{2}$ and $\mathfrak{b}^{2}=\mathfrak{b}_{\max }^{2}$. Thus, $A(\mathrm{H})=\mathfrak{a}^{2}+\mathfrak{b}^{2}=A\left(H_{\max }\right)$. Therefore,

$$
\operatorname{PFSEOWA}\left(H_{11}, H_{12}, \ldots, H_{n m}\right)=H_{\max } \cdot
$$

If $S(H)=S\left(H_{\min }\right)$, then we have $\mathfrak{a}^{2}-\mathfrak{b}^{2}=\mathfrak{a}_{\min }^{2}-\mathfrak{b}_{\min }^{2}$. $\mathfrak{a}^{2}=\mathfrak{a}_{\min }^{2}$ and $\mathfrak{b}^{2}=\mathfrak{b}_{\min }^{2}$.

Thus, $A(H) \mathfrak{a}^{2}+\mathfrak{b}^{2}=\mathfrak{a}_{\min }^{2}+\mathfrak{b}_{\min }^{2}=A\left(H_{\min }\right)$. Therefore,

$$
\operatorname{PFSEOWA}\left(H_{11}, H_{12}, \ldots, H_{n m}\right)=H_{\text {min }} .
$$

So, using (26)-(28), we get

$$
H_{\text {min }} \leq \operatorname{PFSEOWA}\left(H_{11}, H_{12}, \ldots, H_{n m}\right) \leq H_{\max } .
$$

3.1.3. Homogeneity. Prove that PFSEOWA $\left(H_{11}, H_{12}, \ldots, H_{n m}\right)=\partial$, PFSEOWA $\left(H_{11}, H_{12}, \ldots, H_{n m}\right)$ for any positive real number $\partial$.

Proof. Let $H_{i j}$ be a PFSN and $\partial>0$. Then, we know that

$$
\partial H_{i j}=\left\langle\frac{\sqrt{\left(1+\mathfrak{a}^{2}\right)^{\partial}-\left(1-\mathfrak{a}^{2}\right)^{\partial}}}{\sqrt{\left(1+\mathfrak{a}^{2}\right)^{\partial}+\left(1-\mathfrak{a}^{2}\right)^{\partial}}}, \frac{\sqrt{2\left(\mathfrak{b}^{2}\right)^{\partial}}}{\sqrt{\left(2-\mathfrak{b}^{2}\right)^{\partial}+\left(\mathfrak{b}^{2}\right)^{\partial}}}\right\rangle .
$$

So,

$$
\begin{aligned}
& \operatorname{PFSEOWA}\left(H_{11}, H_{12}, \ldots, H_{n m}\right) \\
& =\left\langle\frac{\sqrt{\prod_{j=1}^{m}\left(\prod_{i=1}^{n}\left(1+\mathfrak{a}_{\mathfrak{r}(i) \mathfrak{I}(j)}^{2}\right)^{\mathcal{G}_{i}}\right)^{\mathcal{F}_{j}}-\prod_{j=1}^{m}\left(\prod_{i=1}^{n}\left(1-\mathfrak{a}_{\mathfrak{r}(i) \mathfrak{B}(j)}^{2}\right)^{\mathcal{O}_{i}}\right)^{\mathcal{F}_{j}}}}{\sqrt{\prod_{j=1}^{m}\left(\prod_{i=1}^{n}\left(1+\mathfrak{a}_{\mathfrak{r}(i) \mathfrak{I}(j)}^{2}\right)^{\mathcal{G}_{i}}\right)^{\mathcal{F}_{j}}+\prod_{j=1}^{m}\left(\prod_{i=1}^{n}\left(1-\mathfrak{a}_{\mathfrak{r}(i) \mathfrak{I}(j)}^{2}\right)^{\mathcal{G}_{i}}\right)^{\mathcal{F}_{j}}}}\right. \\
& \left.\frac{\sqrt{2 \prod_{j=1}^{m}\left(\prod_{i=1}^{n}\left(\mathfrak{b}_{\mathfrak{r}(i) \mathfrak{g}(j)}^{2}\right)^{\mathcal{G}_{i}}\right)^{\mathscr{F}_{j}}}}{\sqrt{\prod_{j=1}^{m}\left(\prod_{i=1}^{n}\left(2-\mathfrak{b}_{\mathfrak{r}(i) \mathfrak{g}(j)}^{2}\right)^{\mathcal{G}_{i}}\right)^{\mathscr{F}_{j}}+\prod_{j=1}^{m}\left(\prod_{i=1}^{n}\left(\mathfrak{b}_{\mathfrak{r}(i) \mathfrak{g}(j)}^{2}\right)^{\mathcal{G}_{i}}\right)^{\mathcal{F}_{j}}}}\right\rangle
\end{aligned}
$$




$$
\begin{aligned}
& =\left\langle\frac{\sqrt{\left(\prod_{j=1}^{m}\left(\prod_{i=1}^{n}\left(1+\mathfrak{a}_{\mathfrak{r}(i) \mathfrak{g}(j)}^{2}\right)^{\mathcal{G}_{i}}\right)^{\mathcal{F}_{j}}\right)^{\partial}-\left(\prod_{j=1}^{m}\left(\prod_{i=1}^{n}\left(1-\mathfrak{a}_{\mathfrak{r}(i) \mathfrak{g}(j)}^{2}\right)^{\mathcal{G}_{i}}\right)^{\mathcal{F}_{j}}\right)^{\partial}}}{\sqrt{\left(\prod_{j=1}^{m}\left(\prod_{i=1}^{n}\left(1+\mathfrak{a}_{\mathfrak{r}(i) \mathfrak{s}(j)}^{2}\right)^{\mathcal{G}_{i}}\right)^{\mathcal{F}_{j}}\right)^{\partial}+\left(\prod_{j=1}^{m}\left(\prod_{i=1}^{n}\left(1-\mathfrak{a}_{\mathfrak{r}(i) \mathfrak{g}(j)}^{2}\right)^{\mathscr{G}_{i}}\right)^{\mathcal{F}_{j}}\right)^{\partial}}},\right. \\
& \left.\frac{\sqrt{\left(2 \prod_{j=1}^{m}\left(\prod_{i=1}^{n}\left(\mathfrak{b}_{\mathfrak{r}(i) \mathfrak{s}(j)}^{2}\right)^{\mathcal{G}_{i}}\right)^{\mathcal{F}_{j}}\right)^{\partial}}}{\sqrt{\left(\prod_{j=1}^{m}\left(\prod_{i=1}^{n}\left(2-\mathfrak{b}_{\mathfrak{r}(i) \mathfrak{I}(j)}^{2}\right)^{\mathcal{G}_{i}}\right)^{\mathcal{F}_{j}}\right)^{\partial}}+\left(\prod_{j=1}^{m}\left(\prod_{i=1}^{n}\left(\mathfrak{b}_{\mathfrak{r}(i) \mathfrak{g}(j)}^{2}\right)^{\mathcal{G}_{i}}\right)^{\mathcal{F}_{j}}\right)^{\partial}}\right\rangle \\
& =\partial \operatorname{PFSEOWA}\left(H_{11}, H_{12}, \ldots, H_{n m}\right) \text {. }
\end{aligned}
$$

\section{Proposed Multiattribute Group Decision- Making Approach}

This section has developed a DM approach for solving MAGDM problems based on the proposed PFSEOWA operator and its application.

4.1. Proposed Approach. Let $S=\left\{S^{1}, S^{2}, S^{3}, \ldots, S^{s}\right\}$ be the set of $s$ alternatives, $W=\left\{W_{1}, W_{2}, W_{3}, \ldots, W_{r}\right\}$ be the set of $r$ experts (decision-makers), and $\mathbb{N}=\left\{t_{1}, t_{2}, t_{3}, \ldots, t_{m}\right\}$ be the set of $m$ attributes. Let weighted vector of experts $W(i=$ $1,2,3, \ldots, r)$ be $O=\left(\mathcal{O}_{1}, \mathcal{O}_{2}, \mathcal{O}_{3}, \ldots, \mathcal{O}_{n}\right)^{T}$ such that $\mathcal{O}_{i}>0$, $\sum_{i=1}^{n} \mathcal{O}_{i}=1$. Let weight vector of attributes $t_{i}(i=1,2,3, \ldots, m)$ be $\mathscr{J}=\left(\mathscr{J}_{1}, \mathscr{J}_{2}, \mathscr{J}_{3}, \ldots, \mathscr{J}_{n}\right)^{T}$ such that $\mathscr{J}_{j}>0, \sum_{j=1}^{n} \mathscr{J}_{j}=1$. Team of experts $O_{i}(i=1,2,3, \ldots, r)$ consider the alternatives $S^{i}(i=1,2,3, \ldots, s)$ for attributes in the form of PFSNs such as $F=\left(\mathscr{H}_{i j}\right)_{n * m}=\left(\mathfrak{a}_{i j}, \mathfrak{b}_{i j}\right)_{n * m}$. where $0 \leq \mathfrak{a}_{i j}, \mathfrak{b}_{i j} \leq 1$ and $0 \leq \mathfrak{a}_{i j}^{2}, \mathfrak{b}_{i j}^{2} \leq 1 \forall i, j$ are given in Tables 1-5.

We will apply the proposed PFSEOWA operator to solve the MAGDM problem, which has the following steps:

Step 1. Obtain decision matrices $F=\left(H_{i j}\right)_{n * m}$ in the form of PFSNs for alternatives relative to attributes.

Step 2. Find the associated ordered position matrix by using the score function.

Step 3. By using the normalization formula, normalize the decision matrix to convert the rating value of cost type parameters into benefit type parameters.

$$
M_{i j}=\left\{\begin{array}{l}
\mathscr{H}_{i j}^{c}=\left(b_{i j}, \alpha_{i j}\right) \text { cost type parameter } \\
\mathscr{H}_{i j}=\left(\alpha_{i j}, b\right) \text { benefit type parameter. }
\end{array}\right.
$$

Step 4. Use the developed PFSEOWA operator to aggregate the PFSNs $H_{i j}$ for each alternative $S=\left\{S^{1}, S^{2}, S^{3}, \ldots, S^{s}\right\}$ into the decision matrix $H_{i j}$.

Step 5. Calculate the score values of $H$ for all alternatives by using equation (7).

Step 6. Select the alternative which has the maximum score value and examine the ranking.
4.2. Case Study. The problem of supplier selection is both logically and practically imperative. The best quality supplier is the foundation of supply chain operation management, realizing low-cost efficiency, and an important issue for enterprises. Including eco-friendly elements and other sustainable and progressive features in the supplier selection process makes accurate supplier selection complex and multidimensional. According to the selection of activities that are beneficial to the environment or the society, supplier selection is often referred to as "sustainable supplier selection" in the literature. This is a multifaceted and multidimensional problem with inconsistent standards, and the evaluation process needs to pay attention to multiple perceptions. From these points of view, the issue of supplier selection is often reserved as a "reference" problem in the literature, in which multistandard decision support methods have been widely used. The problem of selecting and evaluating sustainable suppliers has been solved in various tasks. Srivastava [40] proposed intensive stipulations on SSCM, which explains environmental intelligence activity in SSCM, which included construction and material procurance, selection, manufacturing process, packaging, and sanitation items supplied to consumers.

The parable that greening will cause sales to decrease and sophisticated operational expenses has vanished as numerous corporations have now grasped that they will not be able to gratify the customer's craving to combine ecological safety strategies into their desires SSCM and turn it into advanced revenues. There is a connection among a healthier atmosphere where various corporations grow sustainability and economic inducements. Business grown perceptions obsessed by SSCM and determining zones can change how to work to raise revenue. Green logistics can support many diminishing productions such as carbon dioxide and CO. Consumption comprising nonfossil causes of energy alleviates haze that affects our environs irritating to breathe. Quite a lot of measures of fossil fuels have been damaging the atmosphere because of prosperity. For example, for marine life, air travel will also affect adulteration because of diesel engine consumption. In the literature, we explored the aspects for indicating sustainable suppliers consistent with different scientists. In this article, the assortment values for 
TABLE 1: Standards for the selection of the appropriate supplier in SSCM.

\begin{tabular}{ccc}
\hline & Criteria & Definition \\
\hline$t_{1}$ & Quality of service & Rejection ratio, upgrading practice, assurance exposure, and human rights, and advantage guarantee \\
$t_{2}$ & Pollution control & Reducing the waste created and unconstrained in the atmosphere \\
$t_{3}$ & Price & Supplier's market, buyers' market, supply, demand \\
$t_{4}$ & Environmental efficiency & Eco-design stipulations, compounds containing ozone-depleting \\
\hline
\end{tabular}

TABLE 2: PFS decision matrix for $S^{1}$.

\begin{tabular}{ccccc}
\hline & $t_{1}$ & $t_{2}$ & $t_{3}$ & $t_{4}$ \\
\hline$O^{1}$ & $(0.8,0.5)$ & $(0.7,0.5)$ & $(0.6,0.4)$ & $(0.7,0.4)$ \\
$O^{2}$ & $(0.6,0.5)$ & $(0.9,0.1)$ & $(0.7,0.3)$ & $(0.4,0.5)$ \\
$O^{3}$ & $(0.8,0.4)$ & $(0.7,0.5)$ & $(0.6,0.4)$ & $(0.3,0.5)$ \\
$O^{4}$ & $(0.7,0.3)$ & $(0.6,0.5)$ & $(0.4,0.5)$ & $(0.5,0.7)$ \\
\hline
\end{tabular}

TABLE 3: PFS decision matrix for $S^{2}$.

\begin{tabular}{ccccc}
\hline & $t_{1}$ & $t_{2}$ & $t_{3}$ & $t_{4}$ \\
\hline$O^{1}$ & $(0.7,0.5)$ & $(0.8,0.5)$ & $(0.6,0.4)$ & $(0.8,0.4)$ \\
$O^{2}$ & $(0.6,0.3)$ & $(0.9,0.2)$ & $(0.8,0.3)$ & $(0.7,0.5)$ \\
$O^{3}$ & $(0.5,0.4)$ & $(0.6,0.5)$ & $(0.6,0.3)$ & $(0.3,0.6)$ \\
$O^{4}$ & $(0.7,0.4)$ & $(0.6,0.4)$ & $(0.7,0.5)$ & $(0.5,0.7)$ \\
\hline
\end{tabular}

sustainable suppliers that have been considered are given in Table 1.

This example of sustainable supplier selection analyzes papers [41-43] and uses review papers [44-47]. We decided to extract the following four essential criteria. These are $t_{1}$ : quality of service; $t_{2}$ : pollution control; $t_{3}$ : price; $t_{4}$ : environmental efficiency.

Let $\left\{S^{1}, S^{2}, S^{3}, S^{4}, S^{5}\right\}$ be a set of alternatives and $\left\{t_{1}, t_{2}, t_{3}, t_{4}\right\}$ be a set of attributes with the weight vector $\mathscr{J}=(0.2,0.2,0.2,0.4)^{T}$. Here, $t_{1}$ and $t_{3}$ are cost type parameters and $t_{2}$ and $t_{4}$ are benefit type parameters. A team of four experts $O_{r}(r=1,2,3,4)$ with weights $\mathcal{O}=(0.1,0.3,0.3,0.3)^{T}$ decided which supplier is most suitable.

Step 1. According to the expert's opinion, Pythagorean fuzzy soft decision matrices for all alternatives are given in Tables 2-6.

Step 2. Find ordered Pythagorean fuzzy soft decision matrices for all alternatives according to the expert's opinion which are given in Tables 7-11.

Step 3. Because $t_{1}$ and $t_{3}$ are cost type parameters, utilize the normalization formula to obtain normalized ordered Pythagorean fuzzy soft decision matrices which are given in Tables 12-16.

Step 4. Applying the proposed PFSEOWA operator on the acquired data (Tables 12-16), then we get the opinion of decision-makers for alternatives in the form of PFSN such as $H_{1}=\langle 0.5423,0.5345\rangle$, $H_{2}=\langle 0.5601,0.6118\rangle, \quad H_{3}=\langle 0.4930,0.5001\rangle$, $H_{4}=\langle 0.5233,0.6071\rangle$, and $H_{5}=\langle 0.5917,0.5412\rangle$.

Step 5. Use equation (7), $\mathfrak{H}=\alpha_{i j}^{2}-b_{i j}^{2}$ to calculate the score values for all alternatives
TABLE 4: PFS decision matrix for $S^{3}$.

\begin{tabular}{ccccc}
\hline & $t_{1}$ & $t_{2}$ & $t_{3}$ & $t_{4}$ \\
\hline$O^{1}$ & $(0.7,0.5)$ & $(0.7,0.4)$ & $(0.6,0.4)$ & $(0.8,0.4)$ \\
$O^{2}$ & $(0.6,0.6)$ & $(0.9,0.1)$ & $(0.6,0.3)$ & $(0.4,0.5)$ \\
$O^{3}$ & $(0.8,0.3)$ & $(0.7,0.2)$ & $(0.6,0.5)$ & $(0.4,0.5)$ \\
$O^{4}$ & $(0.7,0.6)$ & $(0.3,0.5)$ & $(0.4,0.5)$ & $(0.5,0.6)$ \\
\hline
\end{tabular}

TABle 5: PFS decision matrix for $S^{4}$.

\begin{tabular}{ccccc}
\hline & $t_{1}$ & $t_{2}$ & $t_{3}$ & $t_{4}$ \\
\hline$O^{1}$ & $(0.8,0.5)$ & $(0.7,0.5)$ & $(0.7,0.4)$ & $(0.6,0.4)$ \\
$O^{2}$ & $(0.6,0.4)$ & $(0.8,0.1)$ & $(0.7,0.3)$ & $(0.4,0.7)$ \\
$O^{3}$ & $(0.7,0.4)$ & $(0.7,0.5)$ & $(0.6,0.4)$ & $(0.3,0.5)$ \\
$O^{4}$ & $(0.6,0.3)$ & $(0.6,0.3)$ & $(0.8,0.5)$ & $(0.5,0.6)$ \\
\hline
\end{tabular}

TABle 6: PFS decision matrix for $S^{5}$.

\begin{tabular}{ccccc}
\hline & $t_{1}$ & $t_{2}$ & $t_{3}$ & $t_{4}$ \\
\hline$O^{1}$ & $(0.6,0.5)$ & $(0.6,0.5)$ & $(0.6,0.4)$ & $(0.5,0.4)$ \\
$O^{2}$ & $(0.6,0.4)$ & $(0.8,0.1)$ & $(0.8,0.3)$ & $(0.7,0.5)$ \\
$O^{3}$ & $(0.6,0.4)$ & $(0.7,0.3)$ & $(0.6,0.4)$ & $(0.6,0.5)$ \\
$O^{4}$ & $(0.7,0.4)$ & $(0.7,0.5)$ & $(0.4,0.5)$ & $(0.5,0.8)$ \\
\hline
\end{tabular}

$\mathfrak{H}\left(H_{1}\right)=0.0083, \mathfrak{H}\left(H_{2}\right)=-0.0605, \mathfrak{H}\left(H_{3}\right)=0.0070$ and $\mathfrak{S}\left(H_{4}\right)=-0.0947, \mathfrak{S}\left(H_{5}\right)=0.0572$.

Step 6. After calculation, we get the ranking of alternatives $\mathfrak{H}\left(H_{5}\right)>\mathfrak{H}\left(H_{1}\right)>\mathfrak{H}\left(H_{3}\right)>\mathfrak{H}\left(H_{2}\right)>\mathfrak{M}\left(H_{4}\right)$. So, $S^{5}>S^{1}>S^{3}>S^{2}>S^{4}$.

Hence, the best alternative is $S^{5}$.

\section{Comparative Studies}

To show the effectiveness of the proposed methods, a comparison with some existing techniques offered approach is presented in the following section.

5.1. Comparative Analysis. To verify the effectiveness of the proposed method, a brief comparative analysis has been discussed with some approaches under the considered environment. A summary of all results is given in Table 17. In this work, aggregation operator such as PFSEOWA operators is proposed to fuse evaluation information, and then by using the score function, the ranking of alternatives is evaluated. Furthermore, if only one parameter is supposed rather than one parameter, the PFSS theory reduces to PFS. Thus, PFSS theory is the generalized form of Pythagorean fuzzy set (PFS) theory. Hence, admittedly, the proposed 
TAble 7: Ordered PFS decision matrix for $S^{1}$.

\begin{tabular}{lccll}
\hline & $t_{1}$ & $t_{2}$ & $t_{3}$ & $t_{4}$ \\
\hline$O^{1}$ & $(0.8,0.5)$ & $(0.7,0.5)$ & $(0.6,0.4)$ & $(0.7,0.4)$ \\
$O^{2}$ & $(0.6,0.5)$ & $(0.9,0.1)$ & $(0.7,0.3)$ & $(0.4,0.5)$ \\
$O^{3}$ & $(0.8,0.4)$ & $(0.7,0.5)$ & $(0.6,0.4)$ & $(0.3,0.5)$ \\
$O^{4}$ & $(0.7,0.3)$ & $(0.6,0.5)$ & $(0.4,0.5)$ & $(0.5,0.7)$ \\
\hline
\end{tabular}

TAble 8: Ordered PFS decision matrix for $S^{2}$.

\begin{tabular}{lccll}
\hline & $t_{1}$ & $t_{2}$ & $t_{3}$ & $t_{4}$ \\
\hline$O^{1}$ & $(0.7,0.5)$ & $(0.8,0.5)$ & $(0.6,0.4)$ & $(0.8,0.4)$ \\
$O^{2}$ & $(0.6,0.3)$ & $(0.9,0.2)$ & $(0.8,0.3)$ & $(0.7,0.5)$ \\
$O^{3}$ & $(0.5,0.4)$ & $(0.6,0.5)$ & $(0.6,0.3)$ & $(0.3,0.6)$ \\
$O^{4}$ & $(0.7,0.4)$ & $(0.6,0.4)$ & $(0.7,0.5)$ & $(0.5,0.7)$ \\
\hline
\end{tabular}

TABle 9: Ordered PFS decision matrix for $S^{3}$.

\begin{tabular}{lcccc}
\hline & $t_{1}$ & $t_{2}$ & $t_{3}$ & $t_{4}$ \\
\hline$O^{1}$ & $(0.7,0.5)$ & $(0.7,0.4)$ & $(0.6,0.4)$ & $(0.8,0.4)$ \\
$O^{2}$ & $(0.6,0.6)$ & $(0.9,0.1)$ & $(0.6,0.3)$ & $(0.4,0.5)$ \\
$O^{3}$ & $(0.8,0.3)$ & $(0.7,0.2)$ & $(0.6,0.5)$ & $(0.4,0.5)$ \\
$O^{4}$ & $(0.7,0.6)$ & $(0.3,0.5)$ & $(0.4,0.5)$ & $(0.5,0.6)$ \\
\hline
\end{tabular}

TABle 10: Ordered PFS decision matrix for $S^{4}$.

\begin{tabular}{lcccc}
\hline & $t_{1}$ & $t_{2}$ & $t_{3}$ & $t_{4}$ \\
\hline$O^{1}$ & $(0.8,0.5)$ & $(0.7,0.5)$ & $(0.7,0.4)$ & $(0.6,0.4)$ \\
$O^{2}$ & $(0.6,0.4)$ & $(0.8,0.1)$ & $(0.7,0.3)$ & $(0.4,0.7)$ \\
$O^{3}$ & $(0.7,0.4)$ & $(0.7,0.5)$ & $(0.6,0.4)$ & $(0.3,0.5)$ \\
$O^{4}$ & $(0.6,0.3)$ & $(0.6,0.3)$ & $(0.8,0.5)$ & $(0.5,0.6)$ \\
\hline
\end{tabular}

TABle 11: Ordered PFS decision matrix for $S^{5}$.

\begin{tabular}{lcccc}
\hline & $t_{1}$ & $t_{2}$ & $t_{3}$ & $t_{4}$ \\
\hline$O^{1}$ & $(0.6,0.5)$ & $(0.6,0.5)$ & $(0.6,0.4)$ & $(0.5,0.4)$ \\
$O^{2}$ & $(0.6,0.4)$ & $(0.8,0.1)$ & $(0.8,0.3)$ & $(0.7,0.5)$ \\
$O^{3}$ & $(0.6,0.4)$ & $(0.7,0.3)$ & $(0.6,0.4)$ & $(0.6,0.5)$ \\
$O^{4}$ & $(0.7,0.4)$ & $(0.7,0.5)$ & $(0.4,0.5)$ & $(0.5,0.8)$ \\
\hline
\end{tabular}

TABLE 12: Normalized ordered PFS decision matrix for $S^{1}$.

\begin{tabular}{lcccc}
\hline & $t_{1}$ & $t_{2}$ & $t_{3}$ & $t_{4}$ \\
\hline$O^{1}$ & $(0.5,0.8)$ & $(0.7,0.5)$ & $(0.4,0.6)$ & $(0.7,0.4)$ \\
$O^{2}$ & $(0.5,0.6)$ & $(0.9,0.1)$ & $(0.3,0.7)$ & $(0.4,0.5)$ \\
$O^{3}$ & $(0.4,0.8)$ & $(0.7,0.5)$ & $(0.4,0.6)$ & $(0.3,0.5)$ \\
$O^{4}$ & $(0.3,0.7)$ & $(0.6,0.5)$ & $(0.5,0.4)$ & $(0.5,0.7)$ \\
\hline
\end{tabular}

TABLE 13: Normalized ordered PFS decision matrix for $S^{2}$.

\begin{tabular}{lcccc}
\hline & $t_{1}$ & $t_{2}$ & $t_{3}$ & $t_{4}$ \\
\hline$O^{1}$ & $(0.5,0.7)$ & $(0.8,0.5)$ & $(0.4,0.6)$ & $(0.8,0.4)$ \\
$O^{2}$ & $(0.3,0.6)$ & $(0.9,0.2)$ & $(0.3,0.8)$ & $(0.7,0.5)$ \\
$O^{3}$ & $(0.4,0.5)$ & $(0.6,0.5)$ & $(0.3,0.6)$ & $(0.3,0.6)$ \\
$O^{4}$ & $(0.4,0.7)$ & $(0.6,0.4)$ & $(0.5,0.7)$ & $(0.5,0.7)$ \\
\hline
\end{tabular}


TABLE 14: Normalized ordered PFS decision matrix for $S^{3}$.

\begin{tabular}{lcccc}
\hline & $t_{1}$ & $t_{2}$ & $t_{3}$ & $t_{4}$ \\
\hline$O^{1}$ & $(0.5,0.7)$ & $(0.7,0.4)$ & $(0.4,0.6)$ & $(0.8,0.4)$ \\
$O^{2}$ & $(0.6,0.6)$ & $(0.9,0.1)$ & $(0.3,0.6)$ & $(0.4,0.5)$ \\
$O^{3}$ & $(0.3,0.8)$ & $(0.7,0.2)$ & $(0.5,0.6)$ & $(0.4,0.5)$ \\
$O^{4}$ & $(0.6,0.7)$ & $(0.3,0.5)$ & $(0.5,0.4)$ & $(0.5,0.6)$ \\
\hline
\end{tabular}

TABLE 15: Normalized ordered PFS decision matrix for $S^{4}$.

\begin{tabular}{lcccc}
\hline & $t_{1}$ & $t_{2}$ & $t_{3}$ & $t_{4}$ \\
\hline$O^{1}$ & $(0.5,0.8)$ & $(0.7,0.5)$ & $(0.4,0.7)$ & $(0.6,0.4)$ \\
$O^{2}$ & $(0.4,0.6)$ & $(0.8,0.1)$ & $(0.3,0.7)$ & $(0.4,0.7)$ \\
$O^{3}$ & $(0.4,0.7)$ & $(0.7,0.5)$ & $(0.4,0.6)$ & $(0.3,0.5)$ \\
$O^{4}$ & $(0.3,0.6)$ & $(0.6,0.3)$ & $(0.5,0.8)$ & $(0.5,0.6)$ \\
\hline
\end{tabular}

TABLE 16: Normalized PFS ordered decision matrix for $S^{5}$.

\begin{tabular}{lcccc}
\hline & $t_{1}$ & $t_{2}$ & $t_{3}$ & $t_{4}$ \\
\hline$O^{1}$ & $(0.5,0.6)$ & $(0.6,0.5)$ & $(0.4,0.6)$ & $(0.5,0.4)$ \\
$O^{2}$ & $(0.4,0.6)$ & $(0.8,0.1)$ & $(0.3,0.8)$ & $(0.7,0.5)$ \\
$O^{3}$ & $(0.4,0.6)$ & $(0.7,0.3)$ & $(0.4,0.6)$ & $(0.6,0.5)$ \\
$O^{4}$ & $(0.4,0.7)$ & $(0.7,0.5)$ & $(0.5,0.4)$ & $(0.5,0.8)$ \\
\hline
\end{tabular}

TABLE 17: Comparison of proposed operators with some existing operators.

\begin{tabular}{|c|c|c|c|c|c|c|}
\hline Approach & $S^{1}$ & $S^{2}$ & $S^{3}$ & $S^{4}$ & $S^{5}$ & Alternatives ranking \\
\hline Proposed PFSEOWA operator & 0.0083 & -0.0605 & 0.0070 & -0.0947 & 0.0572 & $S^{5}>S^{1}>S^{3}>S^{2}>S^{4}$ \\
\hline PFSWA [30] & 0.0293 & 0.0369 & 0.0783 & -0.0938 & 0.0858 & $S^{5}>S^{3}>S^{1}>S^{4}>S^{2}$ \\
\hline PFSWG [30] & -0.3306 & -0.1383 & -0.1092 & -0.1661 & -0.5957 & $S^{5}>S^{3}>S^{1}>S^{4}>S^{2}$ \\
\hline PFSEWA [48] & 0.0039 & -0.0376 & 0.0433 & -0.0179 & 0.0644 & $S^{5}>S^{3}>S^{1}>S^{4}>S^{2}$ \\
\hline PFSEWG [49] & -0.4975 & -0.1204 & -0.1775 & -0.0778 & -0.1211 & $S^{5}>S^{2}>S^{4}>S^{3}>S^{1}$ \\
\hline
\end{tabular}

operator in this work is more powerful, reliable, and successful based on the facts mentioned above.

\section{Conclusion}

This manuscript recommends a novel technique for picking sustainable suppliers under PFSS. The deficiency of deliberation of the connection among attributes and ambiguous situations may disturb the inferences of some MAGDM difficulties. The core objective of this research is to develop a PFSEOWA operator to overcome these deficiencies. Moreover, some fundamental properties have been presented, such as idempotency, homogeneity, and boundedness for developed Einstein AO. Furthermore, a DM approach has been established to resolve MAGDM difficulties based on the presented operator. To ensure the strength of the established technique, a comprehensive numerical example has been given to select the sustainable supplier in SSCM. A comparative analysis with some existing approaches is presented. Finally, based on obtained results, it has been concluded that the proposed method in this research is the most feasible and successful method for the MAGDM problem. The future studies will essence on developing more decision-making methods utilizing other operators such as Einstein hybrid geometric and Einstein hybrid averaging operators under the environment of PFSS. We have confidence that nearby these substantial growth and prospects will be helpful to consider world climatecentric organizational research fields.

\section{Data Availability}

No data were used to support this study.

\section{Conflicts of Interest}

The authors declare that they have no conflicts of interest.

\section{References}

[1] L. A. Zadeh, "Fuzzy sets," Information and Control, vol. 8, no. 3, pp. 338-353, 1965.

[2] K. T. Atanassov, "Intuitionistic fuzzy sets," Fuzzy Sets and Systems, vol. 20, no. 1, pp. 87-96, 1986.

[3] Z. Zeshui $\mathrm{Xu}$, "Intuitionistic fuzzy aggregation operators," IEEE Transactions on Fuzzy Systems, vol. 15, no. 6, pp. 1179-1187, 2007.

[4] W. Wang and X. Liu, "Intuitionistic fuzzy geometric aggregation operators based on Einstein operations," International Journal of Intelligent Systems, vol. 26, no. 11, pp. 1049-1075, 2011. 
[5] K. Atanassov and G. Gargov, "Interval valued intuitionistic fuzzy sets," Fuzzy Sets and Systems, vol. 31, no. 3, pp. 343-349, 1989.

[6] R. R. Yager, "Pythagorean membership grades in multicriteria decision making," IEEE Transactions on Fuzzy Systems, vol. 22, no. 4, pp. 958-965, 2013.

[7] K. Rahman, A. Ali, M. Shakeel, M. A. Khan, and M. Ullah, "Pythagorean fuzzy weighted averaging aggregation operator and its application to decision making theory," The Nucleus, vol. 54, no. 3, pp. 190-196, 2017.

[8] D. S. Pamučar and L. M. Savin, "Multiple-criteria model for optimal off-road vehicle selection for passenger transportation: BWM-COPRAS model," Vojnotehnički Glasnik, vol. 68 , no. 1, pp. 28-64, 2020.

[9] K. Rahman, S. Abdullah, A. Ali, and F. Amin, "Pythagorean fuzzy ordered weighted averaging aggregation operator and their application to multiple attribute group decision-making," EURO Journal on Decision Processes, vol. 8, no. 1, pp. 61-77, 2020.

[10] L. Wang and N. Li, "Pythagorean fuzzy interaction power Bonferroni mean aggregation operators in multiple attribute decision making," International Journal of Intelligent Systems, vol. 35, no. 1, pp. 150-183, 2020.

[11] M. Deveci, L. Eriskin, and M. Karatas, "A survey on recent applications of pythagorean fuzzy sets: a state-of-the-art between 2013 and 2020," Pythagorean Fuzzy Sets, pp. 3-38, Springer, Berlin, Germany, 2021.

[12] H. Garg, "Generalised Pythagorean fuzzy geometric interactive aggregation operators using Einstein operations and their application to decision making," Journal of Experimental \& Theoretical Artificial Intelligence, vol. 30, no. 6, pp. 763-794, 2018.

[13] Z. Ali, T. Mahmood, T. Mahmood, K. Ullah, and Q. Khan, "Einstein geometric aggregation operators using a novel complex interval-valued pythagorean fuzzy setting with application in green supplier chain management," Reports in Mechanical Engineering, vol. 2, no. 1, pp. 105-134, 2021.

[14] A. Alosta, O. Elmansuri, and I. Badi, "Resolving a location selection problem by means of an integrated AHP-RAFSI approach," Reports in Mechanical Engineering, vol. 2, no. 1, pp. 135-142, 2021.

[15] T. Milosevic, D. Pamucar, and P. Chatterjee, "Model for selecting a route for the transport of hazardous materials using a fuzzy logic system," Military Technical Courier, vol. 69, no. 2, pp. 355-390, 2021.

[16] D. Molodtsov, "Soft set theory-first results," Computers \& Mathematics with Applications, vol. 37, no. 4-5, pp. 19-31, 1999.

[17] P. K. Maji, R. Biswas, and A. R. Roy, "Soft set theory," Computers \& Mathematics with Applications, vol. 45, no. 4-5, pp. 555-562, 2003.

[18] P. K. Maji, A. R. Roy, and R. Biswas, "An application of soft sets in a decision making problem," Computers \& Mathematics with Applications, vol. 44, no. 8-9, pp. 1077-1083, 2002.

[19] P. K. Maji, R. Biswas, and A. R. Roy, "Intuitionistic fuzzy soft sets," Journal of Fuzzy Mathematics, vol. 9, no. 3, pp. 677-692, 2001.

[20] R. M. Zulqarnain, X. L. Xin, M. Saqlain, and W. A. Khan, "TOPSIS method based on the correlation coefficient of interval-valued intuitionistic fuzzy soft sets and aggregation operators with their application in decision-making," Journal of Mathematics, vol. 2021, Article ID 6656858, 16 pages, 2021.

[21] M. Zulqarnain and F. Dayan, "Selection of best alternative for an automotive company by intuitionistic fuzzy TOPSIS method," International Journal of Scientific \& Technology Research, vol. 6, no. 10, pp. 126-132, 2017.

[22] R. Muhammad Zulqarnain, X. L. Xin, X. Long Xin, and M. Saeed, "Extension of TOPSIS method under intuitionistic fuzzy hypersoft environment based on correlation coefficient and aggregation operators to solve decision making problem," AIMS Mathematics, vol. 6, no. 3, pp. 2732-2755, 2021.

[23] H. Garg and R. Arora, "Generalized intuitionistic fuzzy soft power aggregation operator based on $\mathrm{t}$-norm and their application in multicriteria decision-making," International Journal of Intelligent Systems, vol. 34, no. 2, pp. 215-246, 2019.

[24] X. Peng, Y. Yang, and J. Song, "Pythagoren fuzzy soft set and its application," Computer Engineering, vol. 41, no. 7, pp. 224-229, 2015.

[25] T. M. Athira, S. J. John, S. Jacob John, and H. Garg, "A novel entropy measure of pythagorean fuzzy soft sets," AIMS Mathematics, vol. 5, no. 2, pp. 1050-1061, 2020.

[26] T. M. Athira, S. J. John, and H. Garg, "Entropy and distance measures of pythagorean fuzzy soft sets and their applications," Journal of Intelligent and Fuzzy Systems, vol. 37, no. 3, pp. 4071-4084, 2019.

[27] K. Naeem, M. Riaz, X. Peng, and D. Afzal, "Pythagorean fuzzy soft MCGDM methods based on TOPSIS, VIKOR and aggregation operators," Journal of Intelligent and Fuzzy Systems, vol. 37, no. 5, pp. 6937-6957, 2019.

[28] M. Riaz, K. Naeem, and D. Afzal, "Pythagorean m-polar fuzzy soft sets with TOPSIS method for MCGDM," Punjab University Journal of Mathematics, vol. 52, no. 3, pp. 21-46, 2020.

[29] M. Riaz, N. Khalid, and D. Afzal, "A similarity measure under pythagorean fuzzy soft environment with applications," Computational and Applied Mathematics, vol. 39, no. 4, pp. 1-17, 2020.

[30] R. M. Zulqarnain, X. L. Xin, H. Garg, and W. A. Khan, "Aggregation operators of pythagorean fuzzy soft sets with their application for green supplier chain management," Journal of Intelligent and Fuzzy Systems, vol. 40, no. 3, pp. 5545-5563, 2021.

[31] R. M. Zulqarnain, X. L. Xin, I. Siddique, W. Asghar Khan, and M. A. Yousif, "TOPSIS method based on correlation coefficient under pythagorean fuzzy soft environment and its application towards green supply chain management," Sustainability, vol. 13, Article ID 1642, 2021.

[32] R. M. Zulqarnain, X. L. Xin, H. Garg, and R. Ali, "Interaction aggregation operators to solve multi criteria decision making problem under pythagorean fuzzy soft environment," Journal of Intelligent and Fuzzy Systems, vol. 41, no. 1, pp. 1151-1171, 2021.

[33] I. Siddique, R. M. Zulqarnain, R. Ali, A. Alburaikan, A. Iampan, and A. Hamiden, "A decision-making approach based on score matrix for pythagorean fuzzy soft set," Computational Intelligence and Neuroscience, vol. 2021, Article ID 5447422, 16 pages, 2021.

[34] K. Chatterjee, D. Pamucar, and E. K. Zavadskas, "Evaluating the performance of suppliers based on using the R'AMATELMAIRCA method for green supply chain implementation in electronics industry," Journal of Cleaner Production, vol. 184, pp. 101-129, 2018.

[35] Z. Stevic, D. Pamucar, M. Vasiljevic, G. Stojic, and S. Korica, "Novel integrated multicriteria model for supplier selection: case study construction company," Symmetry, vol. 9, no. 11, Article ID 279, 2017.

[36] G. Stojic, Z. Stevic, J. Antucheviciene, D. Pamucar, and M. Vasiljevic, "A novel rough WASPAS approach for supplier selection in a company manufacturing PVC carpentry products," Information, vol. 9, no. 5, Article ID 121, 2018. 
[37] Z. Stevic, D. Pamucar, A. Puska, and P. Chatterjee, "Sustainable supplier selection in healthcare industries using a new MCDM method: measurement of alternatives and ranking according to COmpromise solution (MARCOS)," Computers \& Industrial Engineering, vol. 140, Article ID 106231, 2020.

[38] H. Garg, "A new generalized Pythagorean fuzzy information aggregation using Einstein operations and its application to decision making," International Journal of Intelligent Systems, vol. 31, no. 9, pp. 886-920, 2016.

[39] H. Garg, "Generalized pythagorean fuzzy geometric aggregation operators using einsteint-norm andt-conorm for multicriteria decision-making process," International Journal of Intelligent Systems, vol. 32, no. 6, pp. 597-630, 2017.

[40] S. K. Srivastava, "Green supply-chain management: a state-ofthe-art literature review," International Journal of Management Reviews, vol. 9, no. 1, pp. 53-80, 2007.

[41] J. Watrobski, W. Sałabun, and G. Ladorucki, "The temporal supplier evaluation model based on multicriteria decision analysis methods," in Proceedings of the Asian Conference on Intelligent Information and Database Systems, pp. 432-442, Kanazawa, Japan, April 2017.

[42] M. Vasiljevic, H. Fazlollahtabar, H. Fazlollahtabar, Ž. Stević, and S. Vesković, "A rough multicriteria approach for evaluation of the supplier criteria in automotive industry," Decision Making: Applications in Management and Engineering, vol. 1, no. 1, pp. 82-96, 2018.

[43] J. Watrobski and W. Sałabun, "Green supplier selection framework based on multicriteria decision-analysis approach," in Proceedings of the International Conference on Sustainable Design and Manufacturing, pp. 361-371, Heraklion, Greece, April 2016.

[44] L. De Boer, E. Labro, and P. Morlacchi, "A review of methods supporting supplier selection," European Journal of Purchasing \& Supply Management, vol. 7, no. 2, pp. 75-89, 2001.

[45] J. Chai, J. N. K. Liu, and E. W. T. Ngai, "Application of decision-making techniques in supplier selection: a systematic review of literature," Expert Systems with Applications, vol. 40, no. 10, pp. 3872-3885, 2013.

[46] K. Govindan, S. Rajendran, J. Sarkis, and P. Murugesan, "Multi criteria decision making approaches for green supplier evaluation and selection: a literature review," Journal of Cleaner Production, vol. 98, pp. 66-83, 2015.

[47] K. Zimmer, M. Fröhling, and F. Schultmann, "Sustainable supplier management-a review of models supporting sustainable supplier selection, monitoring and development," International Journal of Production Research, vol. 54, no. 5, pp. 1412-1442, 2016.

[48] K. Rahman, S. Abdullah, A. Ali, and F. Amin, "Pythagorean fuzzy Einstein hybrid averaging aggregation operator and its application to multiple-attribute group decision making," Journal of Intelligent Systems, vol. 29, no. 1, pp. 736-752, 2020.

[49] K. Rahman, S. Abdullah, S. Abdullah, R. Ahmed, and M. Ullah, "Pythagorean fuzzy Einstein weighted geometric aggregation operator and their application to multiple attribute group decision making," Journal of Intelligent and Fuzzy Systems, vol. 33, no. 1, pp. 635-647, 2017. 